\title{
THE EXISTENCE RESULT OF RENORMALIZED SOLUTION FOR NONLINEAR PARABOLIC SYSTEM WITH VARIABLE EXPONENT AND L $^{1}$ DATA
}

\section{FAIROUZ SOUILAH ${ }^{1}$, MESSAOUD MAOUNI $^{2, *}$ AND KAMEL SLIMANI $^{2}$}

\author{
${ }^{1}$ University 20th August 1955, Skikda, Algeria \\ ${ }^{2}$ Laboratory of Applied Mathematics and History and Didactics of Maths "LAMAHIS", Algeria
}

*Corresponding author: m.maouni@univ-skikda.dz; maouni21@gmail.com

\begin{abstract}
In this paper, we prove the existence result of a renormalized solution to a class of nonlinear parabolic systems, which has a variable exponent Laplacian term and a Leary lions operator with data belong to $L^{1}$.
\end{abstract}

\section{Introduction}

Let $\Omega$ is bounded open domain of $\mathbb{R}^{N},(N \geq 2)$ with lipschiz boundary $\partial \Omega, T$ is a positive number oure aime is to study the existence of renormalized solution for a class of nonlinear parabolic systeme with variable exponent and $L^{1}$ data. More precisely, we study the asymptotic behavrior of the problem

$$
\begin{cases}\left(b_{1}(u)\right)_{t}-\operatorname{div} \mathcal{A}(x, t, \nabla u)+\gamma(u)=f_{1}(x, t, u, v) & \text { in } \quad Q=\Omega \times] 0, T[, \\ \left(b_{2}(v)\right)_{t}-\Delta v+=f_{2}(x, t, u, v) & \text { in } \quad Q=\Omega \times] 0, T[, \\ u=v=0 & \text { on } \quad \Sigma=\partial \Omega \times] 0, T[, \\ b_{1}(u)(t=0)=b_{1}\left(u_{0}\right) & \text { in } \Omega, \\ b_{2}(v)(t=0)=b_{2}\left(v_{0}\right) & \text { in } \Omega,\end{cases}
$$

where $\operatorname{div} \mathcal{A}(x, t, \nabla u)=\operatorname{div}\left(|\nabla u|^{p(x)-2} \nabla u\right)$ is a Leary lions operator (see assumptions (3.1)-(3.3)) with $p: \bar{\Omega} \longrightarrow[1,+\infty)$ be a continuous real-valued function and let $p^{-}=\min _{x \in \bar{\Omega}} p(x)$ and $p^{+}=\max _{x \in \bar{\Omega}} p(x)$

Received January $27^{\text {th }}, 2020$; accepted February $25^{\text {th }}, 2020$; published June $25^{\text {th }}, 2020$.

2010 Mathematics Subject Classification. 35J70, 35D05.

Key words and phrases. nonlinear parabolic systems; variable exponent; renormalized solutions; $L^{1}$ data.

(C)2020 Authors retain the copyrights of their papers, and all open access articles are distributed under the terms of the Creative Commons Attribution License. 
with $1<p^{-} \leq p^{+}<N$. Let $\gamma: \mathbb{R} \rightarrow \mathbb{R}$ with $\gamma(s)=\lambda|s|^{p(x)-2} s$ is a continuous increasing function for $\lambda>0$ and $\gamma(0)=0$ such that $\gamma$ is assumed to belong to $L^{1}(Q)$. The function $f_{i}: Q \times \mathbb{R} \times \mathbb{R} \rightarrow \mathbb{R}$ for $i=$ $\overline{1,2}$ be a Carathéodory function (see assumptions (3.5)-(3.7)).

Finally the function $b: \mathbb{R} \rightarrow \mathbb{R}$ is a strictly increasing $C^{1}$-function lipchizienne with $b_{i}(0)=0$ (see (3.4) ), the data $f_{i}$ and $\left(b_{1}\left(u_{0}\right), b_{2}\left(v_{0}\right)\right)$ is in $\left(L^{1}\right)^{2}$, for $i=\overline{1,2}$.

The study of differential equations and variational problems with nonstandard growth conditions arouses much interest with the development of elastic mechanics, electro-rheological fluid dynamics and image processing, etc ( see [9], [19]) .

Problems of this type have been studied by serval a authors. In $2007 \mathrm{H}$. Redwane, studied the existence of solutions for a class of nonlinear parabolic systems see [18], in 2013 Youssef. B and all studied the existence of a renormalized solution for the nonlinear parabolic systems with unbounded nonlinearities see [2] agin in 2016 B . El Hamdaoui and all in [11] studied the renormalized solutions for nonlinear parabolic systems in the Lebesgue Sobolev Space with variable exponent and $L^{1}$ data. In 2016 [17] authors proved the existence and uniqueness of renormalized solution of a reaction diffusion systems which has a variable exponent Laplacian term and could be applied to image denoising for the case of parabolic equations. In 2010 T. M. Bendahmane, P. Wittbold and A.Zimmermann [7] have proved the existence and uniqueness of renormalized solution to nonlinear parabolic equations with variable exponent and $L^{1}$ data. C. Zhang and S. Zhou studied the renormalized and entropy solution for nonlinear parabolic equation with variable exponent and $L^{1}$ data. Moreover, they obtain the equivalence of renormalized solution and entropy solution(see [23]).

In the present paper we prove the existence of renormalized solution for nonlinear parabolic systems with variable exponent and $L^{1}$ data of (1.1). The notion of renormalized solution was introduced by Diperna and Lions [10] in their study of the Boltzmann equation, and this result can be seen as a generalization of the results obtained by F. Souilah and all in [12].

The paper is organized as follows: Section 2, to recall some basic notations and properties of variable exponent Lebesgue Sobolev space. Section 3 , is devoted to specify the assumptions on, $\mathcal{A}(x, t, \xi), \gamma, b_{1}, b_{2}, f_{1}$, $f_{2}, b_{1}\left(u_{0}\right)$ and $b_{2}\left(v_{0}\right)$ needed in the present study. Section 4 , to give the definition of a renormalized solution of (1.1), and we establish (Theorem (4.1) ) the existence of such a solution.

\section{The Mathematical Preliminaries on Variable Exponent Sobolev Spaces}

In this section, we first recall some results on generalized Lebesgue-Sobolev spaces $L^{p(.)}(\Omega), W^{1, p(.)}(\Omega)$ and $W_{0}^{1, p(.)}(\Omega)$ where $\Omega$ is an open subset of $\mathbb{R}^{N}$. We refer to [13] for further properties of Lebesgue Sobolev spaces with variable exponents. Let $p: \bar{\Omega} \longrightarrow[1,+\infty)$ be a continuous real-valued function and let $p^{-}=\min _{x \in \bar{\Omega}} p(x), p^{+}=\max _{x \in \bar{\Omega}} p(x)$ with $1<p()<$.$N .$ 
2.1. Generalized Lebesgue-Sobolev Spaces. First, denote the variable exponent Lebesgue space $L^{p(\cdot)}(\Omega)$ by

$$
L^{p(.)}(\Omega)=\left\{u \text { measurable function in } \Omega: \rho_{p(.)}(u)=\int_{\Omega}|u|^{p(x)} d x\right\}
$$

which is equipped with the Luxemburg norm

$$
\|u\|_{L^{p(.)}(\Omega)}=\inf \left\{\mu>0, \int_{\Omega}\left|\frac{u(x)}{\mu}\right|^{p(x)} d x \leq 1\right\} .
$$

The space $L^{p(x)}(\Omega)$ is also called a generalized Lebesgue space.

The space $\left(L^{p(.)}(\Omega) ;\|\cdot\|_{p(.)}\right)$ is a separable Banach space. Moreover, if $1<p^{-} \leq p^{+}<+\infty$, then $L^{p(.)}(\Omega)$ is uniformly convex, hence reflexive and its dual space is isomorphic to $L^{p \prime(.)}(\Omega)$, where $\frac{1}{p(x)}+\frac{1}{p^{\prime}(x)}=1$, for $x \in \Omega$.

The following inequality will be used later:

$$
\min \left\{\|u\|_{L^{p(.)}(\Omega)}^{p^{-}},\|u\|_{L^{p(.)}(\Omega)}^{p^{+}}\right\} \leq \int_{\Omega}|u(x)|^{p(x)} d x \leq \max \left\{\|u\|_{L^{p(.)}(\Omega)}^{p^{-}},\|u\|_{L^{p(.)}(\Omega)}^{p^{+}}\right\} .
$$

Finally, the Hölder type inequality

$$
\left|\int_{\Omega} u v d x\right| \leq\left(\frac{1}{p^{-}}+\frac{1}{p^{+}}\right)\|u\|_{p(.)}\|v\|_{p^{\prime}(.)},
$$

for all $u \in L^{p(.)}(\Omega)$ and $v \in L^{p^{\prime}(.)}(\Omega)$.

Next, define the variable exponent Sobolev space $W^{1, p(.)}(\Omega)$ by

$$
W^{1, p(.)}(\Omega)=\left\{u \in L^{p(.)}(\Omega),|\nabla u| \in L^{p(\cdot)}(\Omega)\right\},
$$

which is Banach space equiped with the following norm

$$
\|u\|_{1, p(\cdot)}=\|u\|_{p(.)}+\|\nabla u\|_{p(.)} .
$$

The space $\left(W^{1, p(.)}(\Omega) ;\|\cdot\|_{1, p(.)}\right)$ is a separable and reflexive Banach space. An important role in manipulating the generalized Lebesgue and Sobolev spaces is played by the modular $\rho_{p(.)}$ of the space $L^{p(.)}(\Omega)$. To have the following result:

Proposition 2.1. If $u_{n}, u \in L^{p(.)}(\Omega)$ and $p^{+}<+\infty$, the following properties hold true.

(i) $\|u\|_{p(.)}>1 \Longrightarrow\|u\|_{p(.)}^{p^{+}}<\rho_{p(.)}(u)<\|u\|_{p(.)}^{p^{-}}$,

(ii) $\|u\|_{p(.)}<1 \Longrightarrow\|u\|_{p(.)}^{p^{-}}<\rho_{p(.)}(u)<\|u\|_{p(.)}^{p^{+}}$,

(iii) $\|u\|_{p(.)}<1$ (respectively $\left.=1 ;>1\right) \Longleftrightarrow \rho_{p(.)}(u)<1$ (respectively $=1 ;>1$ ),

(iv) $\left\|u_{n}\right\|_{p(.)} \longrightarrow 0$ (respectively $\left.\longrightarrow+\infty\right) \Longleftrightarrow \rho_{p(.)}\left(u_{n}\right)<1$ (respectively $\longrightarrow+\infty$ ), 
(v) $\rho_{p(.)}\left(\frac{u}{\|u\|_{p(.)}}\right)=1$.

For a measurable function $u: \Omega \longrightarrow \mathbb{R}$, we introduce the following notation

$$
\rho_{1, p(.)}=\int_{\Omega}|u|^{p(x)} d x+\int_{\Omega}|\nabla u|^{p(x)} d x .
$$

Proposition 2.2. If $u \in W^{1, p(.)}(\Omega)$ and $p^{+}<+\infty$, the following properties hold true.

$$
\begin{aligned}
& (i)\|u\|_{1, p(.)}>1 \Longrightarrow\|u\|_{1, p(.)}^{p^{+}}<\rho_{1, p(.)}(u)<\|u\|_{1, p(.)}^{p^{-}}, \\
& (\text {ii })\|u\|_{1, p(.)}<1 \Longrightarrow\|u\|_{1, p(.)}^{p^{-}}<\rho_{1, p(.)}(u)<\|u\|_{1, p(.)}^{p^{+}}, \\
& \text {(iii) } \left.\|u\|_{1, p(.)}<1 \text { (respectively }=1 ;>1\right) \Longleftrightarrow \rho_{1, p(.)}(u)<1 \text { (respectively }=1 ;>1 \text { ). }
\end{aligned}
$$

Extending a variable exponent $p: \bar{\Omega} \longrightarrow[1,+\infty)$ to $\bar{Q}=[0, T] \times \bar{\Omega}$ by setting $p(x, t)=p(x)$ for all $(x, t) \in \bar{Q}$.

We may also consider the generalized Lebesgue space

$$
L^{p(.)}(Q)=\left\{u: Q \longrightarrow \mathbb{R} \text { mesurable such that } \int_{Q}|u(x, t)|^{p(x)} d(x, t)<\infty\right\},
$$

endowed with the norm

$$
\|u\|_{L^{p(.)}(Q)}=\inf \left\{\mu>0, \int_{Q}\left|\frac{u(x, t)}{\mu}\right|^{p(x)} d(x, t) \leq 1\right\},
$$

which share the same properties as $L^{p(.)}(\Omega)$.

\section{The Assumptions on The Data}

This paper, we assume that the following assumptions hold true:

Let $\Omega$ be a bounded open set of $\mathbb{R}^{N}(N \geq 2), T>0$ is given and we set $\left.Q=\Omega \times\right] 0, T\left[\right.$, and $\mathcal{A}: Q \times \mathbb{R}^{N} \rightarrow \mathbb{R}^{N}$ be a Carathéodory function such that for all $\xi, \eta \in \mathbb{R}^{N}, \xi \neq \eta$

$$
\begin{gathered}
\mathcal{A}(x, t, \xi) \cdot \xi \geqslant \alpha|\xi|^{p(x)}, \\
|\mathcal{A}(x, t, \xi)| \leqslant \beta\left[L(x, t)+|\xi|^{p(x)-1}\right], \\
(\mathcal{A}(x, t, \xi)-\mathcal{A}(x, t, \eta)) \cdot(\xi-\eta)>0,
\end{gathered}
$$

where $1<p^{-} \leq p^{+}<+\infty, \alpha, \beta$ are positives constants and $L$ is a nonnegative function in $L^{p^{\prime}(.)}(Q)$, $\gamma: \mathbb{R} \rightarrow \mathbb{R}$ is a continuous increasing function with $\gamma(0)=0$.

Let $b_{i}: \mathbb{R} \rightarrow \mathbb{R}$ is a strictly increasing $C^{1}$-function lipchizienne with $b_{i}(0)=0$ and for any $\rho, \tau$ are positives constants and for $i=\overline{1,2}$ such that

$$
\rho \leq b_{i}^{\prime}(s) \leq \tau, \quad \forall s \in \mathbb{R}
$$


$f_{i}: Q \times \mathbb{R} \times \mathbb{R} \rightarrow \mathbb{R}$ be a Carathéodory function such that for any $k>0$, there exists $\sigma_{k}>0, \quad c_{k} \in L^{1}(Q)$ such that

$$
\left|f_{1}\left(x, t, s_{1}, s_{2}\right)\right| \leq c_{k}(x, t)+\sigma_{k}\left|s_{2}\right|^{2}
$$

for almost every $(x, t) \in(Q)$, for every $s_{1}$ such that $\left|s_{1}\right| \leq k$, and for every $s_{2} \in \mathbb{R}$.

For any $k>0$, there exists $\zeta_{k}>0$ and $G_{k} \in L^{p^{\prime}(.)}(Q)$ such that

$$
\left|f_{2}\left(x, t, s_{1}, s_{2}\right)\right| \leq G_{k}(x, t)+\zeta_{k}\left|s_{1}\right|^{p(x)-1},
$$

for almost every $(x, t) \in(Q)$, for every $s_{2}$ such that $\left|s_{2}\right| \leq k$, and for every $s_{1} \in \mathbb{R}$.

$$
f_{1}\left(x, t, s_{1}, s_{2}\right) s_{1} \geq 0 \text { and } f_{2}\left(x, t, s_{1}, s_{2}\right) s_{2} \geq 0,
$$

$$
\left(b_{1}\left(u_{0}\right), b_{2}\left(v_{0}\right)\right) \in\left(L^{1}(\Omega)\right)^{2} .
$$

\section{The Main Results}

In this section, we study the existence of renormalized solutions to problem (1.1).

Definition 4.1. Let $2-\frac{1}{N+1}<p^{-} \leq p^{+}<N$ and $\left(b_{1}\left(u_{0}\right), b_{2}\left(v_{0}\right)\right) \in\left(L^{1}(\Omega)\right)^{2}$. A measurable functions $(u, v) \in\left(C(] 0, T\left[; L^{1}(\Omega)\right)\right)^{2}$ is a renormalized solution of the problem (1.1) if ,

$$
\begin{aligned}
& T_{k}(u) \in L^{p^{-}}(] 0, T\left[; W_{0}^{1, p(.)}(\Omega)\right), T_{k}(v) \in L^{2}(] 0, T\left[; H_{0}^{1}(\Omega)\right) \text { for any } k>0, \\
& \gamma(u) \in L^{1}(Q) \text { and } f_{i}(x, t, u, v) \in\left(L^{1}(Q)\right)^{2}, \quad \forall i=\overline{1,2}, \\
& \qquad b_{1}(u) \in L^{\infty}(] 0, T\left[; L^{1}(\Omega)\right) \cap L^{q^{-}}(] 0, T\left[; W_{0}^{1, q(.)}(\Omega)\right) \\
& \text { and } \quad b_{2}(v) \in L^{\infty}(] 0, T\left[; L^{1}(\Omega)\right) \cap L^{2}(] 0, T\left[; H_{0}^{1}(\Omega)\right),
\end{aligned}
$$

for all continuous functions $q(x)$ on $\bar{\Omega}$ satisfying $q(x) \in\left[1, p(x)-\frac{N}{N+1}\right)$ for all $x \in \bar{\Omega}$,

$$
\lim _{n \rightarrow \infty} \int_{\{n \leq|u| \leq n+1\}} \mathcal{A}(x, t, \nabla u) \nabla u d x d t+\lim _{n \rightarrow \infty} \int_{\{n \leq|v| \leq n+1\}}|\nabla v|^{2} d x d t=0,
$$

and if, for every function $S \in W^{2, \infty}(\mathbb{R})$ which is piecewise $C^{1}$ and such that $S^{\prime}$ has compact support on $\mathbb{R}$, to have,

$$
\begin{gathered}
\left(B_{S}^{1}(u)\right)_{t}-\operatorname{div}\left(\mathcal{A}(x, t, \nabla u) S^{\prime}(u)\right)+S^{\prime \prime}(u) \mathcal{A}(x, t, \nabla u) \nabla u+\gamma(u) S^{\prime}(u) \\
=f_{1}(x, t, u, v) S^{\prime}(u) \text { in } \mathcal{D}^{\prime}(Q) \\
\left(B_{S}^{2}(v)\right)_{t}-\operatorname{div}\left(\nabla v S^{\prime}(v)\right)+S^{\prime \prime}(v) \nabla v=f_{2}(x, t, u, v) S^{\prime}(v) \text { in } \mathcal{D}^{\prime}(Q)
\end{gathered}
$$




$$
\begin{aligned}
& B_{S}^{1}(u)(t=0)=S\left(b_{1}\left(u_{0}\right)\right) \text { in } \Omega, \\
& B_{S}^{2}(v)(t=0)=S\left(b_{2}\left(v_{0}\right)\right) \text { in } \Omega,
\end{aligned}
$$

where $B_{S}^{i}(z)=\int_{0}^{z} b_{i}^{\prime}(r) S^{\prime}(r) d r$ for $i=\overline{1,2}$.

The following remarks are concerned with a few comments on definition (4.1).

Remark 4.1. Note that, all terms in (4.4) are well defined. Indeed, let $k>0$ such that $\operatorname{supp}\left(S^{\prime}\right) \subset[K, K]$, we have $B_{S}^{i}(u)$ belongs to $L^{\infty}(Q)$ for all $i=\overline{1,2}$ because

$$
\left|B_{S}^{1}(u)\right| \leq \int_{0}^{u}\left|b_{1}^{\prime}(r) S^{\prime}(r)\right| d r \leq \tau\left\|S^{\prime}\right\|_{L^{\infty}(\mathbb{R})},
$$

and

$$
\left|B_{S}^{2}(v)\right| \leq \int_{0}^{v}\left|b_{2}^{\prime}(r) S^{\prime}(r)\right| d r \leq \tau\left\|S^{\prime}\right\|_{L^{\infty}(\mathbb{R})},
$$

and

$$
S(u)=S\left(T_{k}(u)\right) \in L^{p-}(] 0, T\left[; W_{0}^{1, p(\cdot)}(\Omega)\right), S(v)=S\left(T_{k}(v)\right) \in L^{2}(] 0, T\left[; H_{0}^{1}(\Omega)\right)
$$

and $\frac{\partial B_{S}^{i}(u)}{\partial t} \in\left(\mathcal{D}^{\prime}(Q)\right)^{2}$ for $i=\overline{1,2}$. The term $S^{\prime}(u) \mathcal{A}\left(x, t, \nabla T_{k}(u)\right)$ identifes with $S^{\prime}\left(T_{k}(u)\right) \mathcal{A}\left(x, t, \nabla\left(T_{k}(u)\right)\right)$ a.e. in $Q$, where $u=T_{k}(u)$ in $\{|u| \leq k\}$, assumptions (3.2) imply that

$$
\begin{gathered}
\left|S^{\prime}\left(T_{k}(u)\right) \mathcal{A}\left(x, t, \nabla T_{k}(u)\right)\right| \\
\leq \beta\left\|S^{\prime}\right\|_{L^{\infty}(\mathbb{R})}\left[L(x, t)+\left|\nabla\left(T_{k}(u)\right)\right|^{p(x)-1}\right] \text { a.e in } Q .
\end{gathered}
$$

Using (3.2) and (4.1), it follows that $S^{\prime}(u) \mathcal{A}(x, t, \nabla u) \in\left(L^{p^{\prime}(.)}(Q)\right)^{N}$. The term $S^{\prime \prime}(u) \mathcal{A}(x, t, \nabla u) \nabla(u)$ identifes with $S^{\prime \prime}(u) \mathcal{A}\left(t, x, \nabla\left(T_{k}(u)\right)\right) \nabla T_{k}(u)$ and in view of (3.2), (4.1) and (4.8), to obtain $S^{\prime \prime}(u) \mathcal{A}(x, t, \nabla u) \nabla(u) \in L^{1}(Q)$ and $S^{\prime}(u) \gamma(u) \in L^{1}(Q)$. Finally $f_{1}(x, t, u, v) S^{\prime}(u)=f_{1}\left(x, t, T_{k}(u), v\right) S^{\prime}(u)$ a.e in $Q$. Since $\left|T_{k}(u)\right| \leq k$ and $S^{\prime}(u) \in L^{\infty}(Q), c_{k}(x, t) \in L^{1}(Q)$, to obtain from (3.5) that $f_{1}\left(x, t, T_{k}(u), v\right) S^{\prime}(u) \in L^{1}(Q)$, and $f_{2}(x, t, u, v) S^{\prime}(v)=f_{2}\left(x, t, u, T_{k}(v)\right) S^{\prime}(v)$ a.e in $Q$. Since $\left|T_{k}(v)\right| \leq k$ and $S^{\prime}(v) \in L^{\infty}(Q), G_{k}(x, t) \in L^{p^{\prime}(.)}(Q)$, to obtain from (3.6) that $f_{2}\left(x, t, u, T_{k}(v)\right) S^{\prime}(v) \in L^{1}(Q)$. Also $\frac{\partial B_{S}^{1}(u)}{\partial t} \in L^{\left(p^{-}\right)^{\prime}}(] 0, T\left[; W^{-1, p^{\prime}(.)}(\Omega)\right)+L^{1}(Q)$ and $B_{S}^{1}(u) \in L^{p^{-}}(] 0, T\left[; W_{0}^{1, p(.)}(\Omega)\right) \cap L^{\infty}(Q)$, and $\frac{\partial B_{S}^{2}(v)}{\partial t} \in$ $L^{2}(] 0, T\left[; H^{-1}(\Omega)\right)+L^{1}(Q)$ and $B_{S}^{2}(v) \in L^{2}(] 0, T\left[; H_{0}^{1}(\Omega)\right) \cap L^{\infty}(Q)$, which implies that $\left(B_{S}^{1}(u), B_{S}^{2}(v)\right)$ $\in\left(C(] 0, T\left[; L^{1}(\Omega)\right)\right)^{2}$. 


\subsection{The Existence Theorem.}

Theorem 4.1. Let $\left(b_{1}\left(u_{0}\right), b_{2}\left(v_{0}\right)\right) \in\left(L^{1}(\Omega)\right)^{2}$, assume that (3.1)-(3.8) hold true, then there exists at least one renormalized solution $(u, v) \in\left(C(] 0, T\left[, L^{1}(\Omega)\right)\right)^{2}$ of Problem (1.1) ( in the sens of Definition (4.1)).

Proof. of Theorem (4.1) The above theorem is to be proved in five steps.

- Step 1: Approximate problem and a priori estimates. Let us define the following approximation of $b$ and $f$ for $\varepsilon>0$ fixed and for $i=\overline{1,2}$

$$
b_{\varepsilon}^{i}(r)=T_{\frac{1}{\varepsilon}}\left(b_{i}(r)\right) \text { a.e in } \Omega \text { for } \varepsilon>0, \quad \forall r \in \mathbb{R},
$$

$b_{\varepsilon}^{i}\left(u_{0}^{\varepsilon}\right)$ are a sequence of $\left(C_{c}^{\infty}(\Omega)\right)^{2}$ functions such that

$$
\left(b_{\varepsilon}^{1}\left(u_{0}^{\varepsilon}\right), b_{\varepsilon}^{2}\left(v_{0}^{\varepsilon}\right)\right) \rightarrow\left(b_{1}\left(u_{0}\right), b_{2}\left(v_{0}\right)\right) \text { in }\left(L^{1}(\Omega)\right)^{2} \text { as } \varepsilon \text { tends to } 0 .
$$

$$
\begin{aligned}
& f_{1}^{\varepsilon}\left(x, t, r_{1}, r_{2}\right)=f_{1}\left(x, t, T_{\frac{1}{\varepsilon}}\left(r_{1}\right), r_{2}\right), \\
& f_{2}^{\varepsilon}\left(x, t, r_{1}, r_{2}\right)=f_{2}\left(x, t, r_{1}, T_{\frac{1}{\varepsilon}}\left(r_{2}\right)\right),
\end{aligned}
$$

in view of (3.5), (3.6) and (3.7), there exist $G_{k}^{\varepsilon} \in L^{p^{\prime}(.)}(Q), c_{k}^{\varepsilon} \in L^{1}(Q)$ and $\sigma_{k}^{\varepsilon}, \zeta_{k}^{\varepsilon}>0$ such that

$$
\begin{gathered}
\left|f_{1}^{\varepsilon}\left(x, t, s_{1}, s_{2}\right)\right| \leq c_{k}^{\varepsilon}(x, t)+\sigma_{k}^{\varepsilon}\left|s_{2}\right|^{2}, \\
\left|f_{2}^{\varepsilon}\left(x, t, s_{1}, s_{2}\right)\right| \leq G_{k}^{\varepsilon}(x, t)+\zeta_{k}^{\varepsilon}\left|s_{1}\right|^{p(x)-1},
\end{gathered}
$$

for almost every $(x, t) \in(Q), s_{1}, s_{2} \in \mathbb{R}$,

$$
f_{1}^{\varepsilon}\left(x, t, s_{1}, s_{2}\right) s_{1} \geq 0 \text { and } f_{2}^{\varepsilon}\left(x, t, s_{1}, s_{2}\right) s_{2} \geq 0 .
$$

Let us now consider the approximate problem:

$$
\begin{gathered}
\left(b_{\varepsilon}^{1}\left(u^{\varepsilon}\right)\right)_{t}-\operatorname{div} \mathcal{A}\left(x, t, \nabla u^{\varepsilon}\right)+\gamma\left(u^{\varepsilon}\right)=f_{1}^{\varepsilon}\left(x, t, u^{\varepsilon}, v^{\varepsilon}\right) \text { in } Q, \\
\left(b_{\varepsilon}^{2}\left(v^{\varepsilon}\right)\right)_{t}-\Delta v^{\varepsilon}=f_{2}^{\varepsilon}\left(x, t, u^{\varepsilon}, v^{\varepsilon}\right) \text { in } Q, \\
\left.u^{\varepsilon}=v^{\varepsilon}=0 \text { on }\right] 0, T[\times \partial \Omega, \\
b_{\varepsilon}^{1}\left(u^{\varepsilon}\right)(t=0)=b_{\varepsilon}^{1}\left(u_{0}^{\varepsilon}\right) \text { in } \Omega, \\
b_{\varepsilon}^{2}\left(v^{\varepsilon}\right)(t=0)=b_{\varepsilon}^{2}\left(v_{0}^{\varepsilon}\right) \text { in } \Omega .
\end{gathered}
$$


As a consequence, proving existence of a weak solution $u^{\varepsilon} \in L^{p^{-}}(] 0, T\left[; W_{0}^{1, p(.)}(\Omega)\right)$ and $v^{\varepsilon} \in$ $L^{2}(] 0, T\left[; H_{0}^{1}(\Omega)\right)$ of (4.15)-(4.18) is an easy task (see [15]).

we choose $T_{k}\left(u^{\varepsilon}\right) \chi_{(0, t)}$ as a test function in (4.15), to get

$$
\begin{aligned}
\int_{\Omega} B_{k}^{1, \varepsilon}\left(u^{\varepsilon}\right)(t) d x & +\int_{0}^{t} \int_{\Omega} \mathcal{A}\left(x, t, \nabla u^{\varepsilon}\right) \nabla T_{k}\left(u^{\varepsilon}\right)+\int_{0}^{t} \int_{\Omega} \gamma\left(u^{\varepsilon}\right) T_{k}\left(u^{\varepsilon}\right) d x d s \\
& =\int_{0}^{t} \int_{\Omega} f_{1}^{\varepsilon}\left(x, t, u^{\varepsilon}, v^{\varepsilon}\right) T_{k}\left(u^{\varepsilon}\right) d x d s+\int_{\Omega} B_{k}^{1, \varepsilon}\left(u_{0}^{\varepsilon}\right) d x
\end{aligned}
$$

for almost every $t$ in $(0, T)$, and where

$$
B_{k}^{i, \varepsilon}(r)=\int_{0}^{r} T_{k}(s) \frac{\partial b_{\varepsilon}^{i}(s)}{\partial s} d s . \forall i=\overline{1,2} .
$$

Under the definition of $B_{k}^{i, \varepsilon}(r)$ the inequality

$$
0 \leq \int_{\Omega} B_{k}^{1, \varepsilon}\left(u_{0}^{\varepsilon}\right)(t) d x \leq k \int_{\Omega}\left|b_{\varepsilon}^{1}\left(u_{0}^{\varepsilon}\right)\right| d x, \quad k>0 .
$$

Using (3.1), $f_{1}^{\varepsilon}\left(x, t, u^{\varepsilon}, v^{\varepsilon}\right) T_{k}\left(u^{\varepsilon}\right) \geq 0$, and we have $\gamma\left(u^{\varepsilon}\right)=\lambda\left|u^{\varepsilon}\right|^{p(x)-1} u^{\varepsilon} \geq 0$ because $1<p^{-} \leq$ $p(x) \leq+\infty$ and the definition of $B_{k}^{\varepsilon}(r)$ in (4.20), to obtain

$$
\int_{\Omega} B_{k}^{\varepsilon}\left(u^{1, \varepsilon}\right)(t) d x+\alpha \int_{E_{k}}\left|\nabla u^{\varepsilon}\right|^{p(x)} d x d s \leq k\left\|b_{\varepsilon}^{1}\left(u_{0}^{\varepsilon}\right)\right\|_{L^{1}(Q)},
$$

where $E_{k}=\left\{(x, t) \in Q:\left|u^{\varepsilon}\right| \leq k\right\}$, using $\bar{B}_{k}^{\varepsilon}\left(u^{\varepsilon}\right)(t) \geq 0$ and inequality (2.2) in (4.21), to get

$$
\begin{aligned}
\alpha \int_{0}^{T} \min \left\{\left\|\nabla T_{k}\left(u^{\varepsilon}\right)\right\|_{L^{p(x)}(\Omega)}^{p^{-}},\left\|\nabla T_{k}\left(u^{\varepsilon}\right)\right\|_{L^{p(x)}(\Omega)}^{p^{+}}\right\} & \leq \alpha \int_{\left\{(x, t) \in Q:\left|u^{\varepsilon}\right| \leq k\right\}}\left|\nabla u^{\varepsilon}\right|^{p(x)} d x d t \\
& \leq C,
\end{aligned}
$$

then is $T_{k}\left(u^{\varepsilon}\right)$ is bounded in $L^{p-}(] 0, T\left[; W_{0}^{1, p(x)}(\Omega)\right)$.

Similarly, we choose $T_{k}\left(v^{\varepsilon}\right) \chi_{(0, t)}$ as a test function in (4.16), to get

$$
\int_{\Omega} B_{k}^{2, \varepsilon}\left(v^{\varepsilon}\right)(t) d x+\alpha \int_{F_{k}}\left|\nabla v^{\varepsilon}\right|^{2} d x d s \leq k\left\|b_{\varepsilon}^{2}\left(v_{0}^{\varepsilon}\right)\right\|_{L^{1}(Q)},
$$

where $F_{k}=\left\{(x, t) \in Q:\left|v^{\varepsilon}\right| \leq k\right\}$, then is $T_{k}\left(v^{\varepsilon}\right)$ is bounded in $L^{2}(] 0, T\left[; H_{0}^{1}(\Omega)\right)$. Adding (4.21) and (4.23), one gets

$$
\int_{\Omega} B_{k}^{1, \varepsilon}\left(u^{\varepsilon}\right)(t) d x+\int_{\Omega} B_{k}^{2, \varepsilon}\left(v^{\varepsilon}\right)(t) d x \leq k\left\|\left(b_{\varepsilon}^{1}\left(u_{0}^{\varepsilon}\right), b_{\varepsilon}^{2}\left(v_{0}^{\varepsilon}\right)\right)\right\|_{L^{1}(Q) \times L^{1}(Q)} .
$$

Also, to obtain

$$
k \int_{\left\{(t, x) \in Q:\left|u^{\varepsilon}\right|>k\right\}}\left|\gamma\left(u^{\varepsilon}\right)\right| d x d t \leq k\left\|b_{\varepsilon}\left(u_{0}^{\varepsilon}\right)\right\|_{L^{1}(Q)} .
$$


Hence

$$
\begin{aligned}
k \int_{\left\{(x, t) \in Q:\left|u^{\varepsilon}\right|>k\right\}}\left|f_{1}^{\varepsilon}\left(x, t, u^{\varepsilon}, v^{\varepsilon}\right)\right| d x d t+ & k \int_{\left\{(x, t) \in Q:\left|v^{\varepsilon}\right|>k\right\}}\left|f_{2}^{\varepsilon}\left(x, t, u^{\varepsilon}, v^{\varepsilon}\right)\right| d x d t \\
& \leq k\left\|\left(b_{\varepsilon}^{1}\left(u_{0}^{\varepsilon}\right), b_{\varepsilon}^{2}\left(v_{0}^{\varepsilon}\right)\right)\right\|_{L^{1}(Q) \times L^{1}(Q)} .
\end{aligned}
$$

Now, let $T_{1}\left(s-T_{k}(s)\right)=T_{k, 1}(s)$ and take $T_{k, 1}\left(b_{\varepsilon}^{1}\left(u^{\varepsilon}\right)\right)$ as test function in (4.15). Reasoning as above, by $\nabla T_{k, 1}(s)=\nabla s \chi_{\{k \leq|s| \leq k+1\}}$ and the young's inequality, to obtain

$$
\begin{aligned}
\alpha \int_{\left\{k \leq\left|b_{\varepsilon}^{1}\left(u^{\varepsilon}\right)\right| \leq k+1\right\}} b_{1, \varepsilon}^{\prime}\left(u^{\varepsilon}\right)\left|\nabla\left(u^{\varepsilon}\right)\right|^{p(x)} d x d t & \leq k \int_{\left\{\left|b_{\varepsilon}^{1}\left(u_{0}^{\varepsilon}\right)\right|>k\right\}}\left|b_{\varepsilon}^{1}\left(u_{0}^{\varepsilon}\right)\right| d x \\
& +C k \int_{\left\{\left|b_{\varepsilon}^{1}\left(u^{\varepsilon}\right)\right|>k\right\}}\left|\gamma\left(u^{\varepsilon}\right)\right| d x d t \\
& +C k \int_{\left\{\left|b_{\varepsilon}^{1}\left(u^{\varepsilon}\right)\right|>k\right\}}\left|f_{1}^{\varepsilon}\left(x, t, u^{\varepsilon}, v^{\varepsilon}\right)\right| d x d t \\
& \leq C_{1},
\end{aligned}
$$

inequality (2.2) implies that

$$
\begin{aligned}
& \int_{0}^{T} \alpha \chi_{\left\{k \leq\left|b_{\varepsilon}^{1}\left(u^{\varepsilon}\right)\right| \leq k+1\right\}} \min \left\{\left\|\nabla\left(b_{\varepsilon}^{1}\left(u^{\varepsilon}\right)\right)\right\|_{L^{p(x)}(\Omega)}^{p-},\left\|\nabla\left(b_{\varepsilon}^{1}\left(u^{\varepsilon}\right)\right)\right\|_{L^{p(x)}(\Omega)}^{p+}\right\} \\
& \leq \alpha \int_{\left\{k \leq\left|b_{\varepsilon}^{1}\left(u^{\varepsilon}\right)\right| \leq k+1\right\}} b_{1, \varepsilon}^{\prime}\left(u^{\varepsilon}\right)\left|\nabla\left(u^{\varepsilon}\right)\right|^{p(x)} d x d t \leq C_{1} .
\end{aligned}
$$

Similarly, we choose $T_{k}\left(b_{\varepsilon}^{2}\left(v^{\varepsilon}\right)\right)$ as test function in (4.16), to have

$$
\begin{aligned}
\int_{\left\{\left|b_{\varepsilon}^{2}\left(v^{\varepsilon}\right)\right| \leq k\right\}} b_{2, \varepsilon}^{\prime}\left(v^{\varepsilon}\right)\left|\nabla\left(v^{\varepsilon}\right)\right|^{2} d x d t \leq & k \int_{\left\{\left|b_{\varepsilon}^{2}\left(v_{0}^{\varepsilon}\right)\right|>k\right\}}\left|b_{\varepsilon}^{2}\left(v_{0}^{\varepsilon}\right)\right| d x \\
& +C k \int_{\left\{\left|b_{\varepsilon}^{2}\left(v^{\varepsilon}\right)\right|>k\right\}}\left|f_{2}^{\varepsilon}\left(x, t, u^{\varepsilon}, v^{\varepsilon}\right)\right| d x d t \leq C_{2},
\end{aligned}
$$

we know that properties of $B_{k}^{i, \varepsilon}\left(u^{\varepsilon}\right), \quad\left(B_{k}^{i, \varepsilon}\left(r^{\varepsilon}\right) \geq 0, \quad B_{k}^{i, \varepsilon}\left(r^{\varepsilon}\right)\right) \geq \rho(|r|-1), \quad$ for all $i=\overline{1,2}$, to obtain

$$
\begin{aligned}
\int_{\Omega}\left|B_{k}^{1, \varepsilon}\left(u^{\varepsilon}\right)(t)\right| d x & +\int_{\Omega}\left|B_{k}^{2, \varepsilon}\left(v^{\varepsilon}\right)(t)\right| d x \leq k \int_{\Omega}\left|b_{\varepsilon}^{1}\left(u^{\varepsilon}\right)(t)\right| d x+k \int_{\Omega}\left|b_{\varepsilon}^{2}\left(v^{\varepsilon}\right)(t)\right| d x \\
& \leq \rho\left(2 \operatorname{meas}(\Omega)+k\left\|\left(b_{\varepsilon}^{1}\left(u_{0}^{\varepsilon}\right), b_{\varepsilon}^{2}\left(v_{0}^{\varepsilon}\right)\right)\right\|_{L^{1}(Q) \times L^{1}(Q)}\right) .
\end{aligned}
$$

From the estimation $(4.22),(4.23),(4.27),(4.28)$ and the properites of $B_{k}^{i, \varepsilon}$ and $b_{\varepsilon}^{1}\left(u_{0}^{\varepsilon}\right), b_{\varepsilon}^{2}\left(v_{0}^{\varepsilon}\right)$, we deduce that

$$
b_{\varepsilon}^{1}\left(u^{\varepsilon}\right) \text { and } b_{\varepsilon}^{2}\left(v^{\varepsilon}\right) \text { is bounded in } L^{\infty}(] 0, T\left[; L^{1}(\Omega)\right)
$$

$$
u^{\varepsilon} \text { and } v^{\varepsilon} \text { is bounded in } L^{\infty}(] 0, T\left[; L^{1}(\Omega)\right)
$$


and

$$
b_{\varepsilon}^{1}\left(u^{\varepsilon}\right) \text { is bounded in } L^{p-}(] 0, T\left[; W_{0}^{1, p(x)}(\Omega)\right),
$$

and

$$
b_{\varepsilon}^{2}\left(v^{\varepsilon}\right) \text { is bounded in } L^{2}(] 0, T\left[; H_{0}^{1}(\Omega)\right),
$$

by $(4.27),(4.28)$ and Lemma 2.1 in [7] by and if

$$
2-\frac{1}{N+1}<p(.)<N,
$$

to obtain

$$
b_{\varepsilon}^{1}\left(u^{\varepsilon}\right) \text { is bounded in } L^{q-}(] 0, T\left[; W_{0}^{1, q(x)}(\Omega)\right),
$$

for all continuous variable exponents $q \in C(\bar{\Omega})$ satisfying

$$
1 \leq q(x)<\frac{N(p(x)-1)+p(x)}{N+1},
$$

for all $x \in \Omega$.

And

$$
T_{k}\left(u^{\varepsilon}\right) \text { is bounded in } L^{p^{-}}(] 0, T\left[; W_{0}^{1, p(\cdot)}(\Omega)\right)
$$

and

$$
T_{k}\left(v^{\varepsilon}\right) \text { is bounded in } L^{2}(] 0, T\left[; H_{0}^{1}(\Omega)\right) .
$$

By (4.25) and (4.26), we may conclude that

$$
\gamma\left(u^{\varepsilon}\right) \text { is bounded in } L^{1}(] 0, T\left[; L^{1}(\Omega)\right)
$$

and

$$
f_{1}^{\varepsilon}\left(x, t, u^{\varepsilon}, v^{\varepsilon}\right) \text { and } f_{2}^{\varepsilon}\left(x, t, u^{\varepsilon}, v^{\varepsilon}\right) \text { is bounded in } L^{1}(] 0, T\left[; L^{1}(\Omega)\right) \text {, }
$$

independently of $\varepsilon$.

Proceeding as in [3], [4] that for any $S \in W^{2, \infty}(\mathbb{R})$ such that $S^{\prime}$ is compact (supp $S^{\prime} \subset[-k, k]$ ),

$$
S\left(u^{\varepsilon}\right) \text { is bounded in } L^{p-}(] 0, T\left[; W_{0}^{1, p(.)}(\Omega)\right),
$$

and

$$
S\left(v^{\varepsilon}\right) \text { is bounded in } L^{2}(] 0, T\left[; H_{0}^{1}(\Omega)\right),
$$

and

$$
\left(S\left(u^{\varepsilon}\right)\right)_{t} \text { is bounded in } L^{1}(Q)+L^{(p-)^{\prime}}(] 0, T\left[; W^{-1, p^{\prime}(.)}(\Omega)\right)
$$

and

$$
\left(S\left(v^{\varepsilon}\right)\right)_{t} \text { is bounded in } L^{1}(Q)+L^{2}(] 0, T\left[; H^{-1}(\Omega)\right) .
$$


In fact, as a consequence of (4.34), by Stampacchia's Theorem, we obtain (4.38). To show that (4.40) holds true, we multiply the equation (4.15) by $S^{\prime}\left(u^{\varepsilon}\right)$ and the equation (4.16) by $S^{\prime}\left(v^{\varepsilon}\right)$, to obtain

$$
\begin{gathered}
\left(B_{S}^{1}\left(u^{\varepsilon}\right)\right)_{t}=\operatorname{div}\left(S^{\prime}\left(u^{\varepsilon}\right) \mathcal{A}\left(x, t, \nabla u^{\varepsilon}\right)\right)-\mathcal{A}\left(x, t, \nabla u^{\varepsilon}\right) \nabla\left(S^{\prime}\left(u^{\varepsilon}\right)\right) \\
-\gamma\left(u^{\varepsilon}\right) S^{\prime}\left(u^{\varepsilon}\right)+f_{1}^{\varepsilon}\left(x, t, u^{\varepsilon}, v^{\varepsilon}\right) S^{\prime}\left(u^{\varepsilon}\right) \text { in } \mathcal{D}^{\prime}(Q)
\end{gathered}
$$

And

$$
\begin{gathered}
\left(B_{S}^{2}\left(v^{\varepsilon}\right)\right)_{t}=\operatorname{div}\left(S^{\prime}\left(v^{\varepsilon}\right) \nabla v^{\varepsilon}\right)-\nabla\left(S^{\prime}\left(v^{\varepsilon}\right)\right) \\
+f_{2}^{\varepsilon}\left(x, t, u^{\varepsilon}, v^{\varepsilon}\right) S^{\prime}\left(v^{\varepsilon}\right) \text { in } \mathcal{D}^{\prime}(Q) .
\end{gathered}
$$

Since $\operatorname{supp}\left(S^{\prime}\right)$ and $\operatorname{supp}\left(S^{\prime \prime}\right)$ are both included in $[-k ; k] ; u^{\varepsilon}$ may be replaced by $T_{k}\left(u^{\varepsilon}\right)$ in $\left\{\left|u^{\varepsilon}\right| \leq k\right\}$. To have

$$
\begin{gathered}
\left|S^{\prime}\left(u^{\varepsilon}\right) \mathcal{A}\left(x, t, \nabla u^{\varepsilon}\right)\right| \\
\leq \beta\left\|S^{\prime}\right\|_{L^{\infty}}\left[L(x, t)+\left|\nabla T_{k}\left(u^{\varepsilon}\right)\right|^{p(x)-1}\right],
\end{gathered}
$$

as a consequence, each term in the right hand side of (4.42) is bounded either in $L^{(p-)^{\prime}}(] 0, T\left[; W^{-1, p^{\prime}(.)}(\Omega)\right)$ or in $L^{1}(Q)$, and obtain (4.40).

Now we look for an estimate on a sort of energy at infinity of the approximating solutions. For any integer $n \geq 1$, consider the Lipschitz continuous function $\theta_{n}$ defined through

$$
\theta_{n}(s)=T_{n+1}(s)-T_{n}(s)=\left\{\begin{array}{cl}
0 & \text { if }|s| \leq n, \\
(|s|-n) \operatorname{sign}(s) & \text { if } n \leq|s| \leq n+1, \\
\operatorname{sign}(s) & \text { if }|s| \geq n
\end{array}\right.
$$

Remark that $\left\|\theta_{n}\right\|_{L^{\infty}} \leq 1$ for any $n \geq 1$ and that $\theta_{n}(s) \rightarrow 0$, for any $s$ when $n$ tends to infinity. Using the admissible test function $\theta_{n}\left(u^{\varepsilon}\right)$ in (4.15) leads to

$$
\begin{aligned}
\int_{\Omega} \widetilde{\theta_{n}}\left(u^{\varepsilon}\right)(t) d x & +\int_{Q} \mathcal{A}\left(x, t, \nabla u^{\varepsilon}\right) \nabla\left(\theta_{n}\left(u^{\varepsilon}\right)\right) d x d t+\int_{Q} \gamma\left(u^{\varepsilon}\right) \theta_{n}\left(u^{\varepsilon}\right) d x d t \\
& =\int_{Q} f^{\varepsilon}\left(x, t, u^{\varepsilon}\right) \theta_{n}\left(u^{\varepsilon}\right) d x d t+\int_{\Omega} \widetilde{\theta_{n}}\left(u_{0}^{\varepsilon}\right) d x
\end{aligned}
$$

where $\widetilde{\theta_{n}}(r)(t)=\int_{0}^{r} \theta_{n}(s) \frac{\partial b_{\varepsilon}^{i}(s)}{\partial s} d s$, for all $i=\overline{1,2}$,

for almost any $t$ in $] 0, T\left[\right.$ and where $\widetilde{\theta_{n}}(r)=\int_{0}^{r} \theta_{n}(s) d s \geq 0$. Hence, dropping a nonnegative term

$$
\int_{\left\{n \leq\left|u^{\varepsilon}\right| \leq n+1\right\}} \mathcal{A}\left(x, t, \nabla u^{\varepsilon}\right) \nabla u^{\varepsilon} d x d t
$$




$$
\begin{aligned}
& \leq \int_{Q} \gamma\left(u^{\varepsilon}\right) \theta_{n}\left(u^{\varepsilon}\right) d x d t+\int_{Q} f_{1}^{\varepsilon}\left(x, t, u^{\varepsilon}, v^{\varepsilon}\right) \theta_{n}\left(u^{\varepsilon}\right) d x d t+\int_{\Omega} \widetilde{\theta_{n}}\left(u_{0}^{\varepsilon}\right) d x \\
& \leq \int_{\left\{\left|u^{\varepsilon}\right| \geq n\right\}}\left|\gamma\left(u^{\varepsilon}\right)\right| d x d t+\int_{\left\{\left|u^{\varepsilon}\right| \geq n\right\}}\left|f_{1}^{\varepsilon}\left(x, t, u^{\varepsilon}, v^{\varepsilon}\right)\right| d x d t+\int_{\left\{\left|b_{\varepsilon}^{1}\left(u_{0}^{\varepsilon}\right)\right| \geq n\right\}}\left|b_{\varepsilon}^{1}\left(u_{0}^{\varepsilon}\right)\right| d x .
\end{aligned}
$$

Similarly, we take test function $\theta_{n}\left(v^{\varepsilon}\right)$ in (4.16) leads to

$$
\begin{gathered}
\int_{\left\{n \leq\left|v^{\varepsilon}\right| \leq n+1\right\}}\left|\nabla v^{\varepsilon}\right|^{2} d x d t \\
\leq \int_{Q} f_{2}^{\varepsilon}\left(x, t, u^{\varepsilon}, v^{\varepsilon}\right) \theta_{n}\left(v^{\varepsilon}\right) d x d t+\int_{\Omega} \widetilde{\theta_{n}}\left(v_{0}^{\varepsilon}\right) d x \leq \int_{\left\{\left|v^{\varepsilon}\right| \geq n\right\}}\left|f_{2}^{\varepsilon}\left(x, t, u^{\varepsilon}, v^{\varepsilon}\right)\right| d x d t \\
+\int_{\left\{\left|b_{\varepsilon}^{2}\left(v_{0}^{\varepsilon}\right)\right| \geq n\right\}}\left|b_{\varepsilon}^{2}\left(v_{0}^{\varepsilon}\right)\right| d x .
\end{gathered}
$$

Next, we study the convergence of $\left(u_{n}\right)_{n \in \mathbb{N}}$ and $\left(v_{n}\right)_{n \in \mathbb{N}}$ in $C(] 0, T\left[; L^{1}(\Omega)\right)$.

Lemma 4.1. Both $\left(u^{\varepsilon_{n}}\right)_{n \in \mathbb{N}}$ and $\left(v^{\varepsilon_{n}}\right)_{n \in \mathbb{N}}$ are Cauchy sequences in $C(] 0, T\left[; L^{1}(\Omega)\right)$.

Proof. Let $\varepsilon_{n}$ and $\varepsilon_{m}$ two positive integers. It follows frome (4.15) and (4.16) that

$$
\begin{aligned}
& \int_{\Omega} \frac{\partial b_{\varepsilon_{n}}^{1}\left(u^{\varepsilon_{n}}-u^{\varepsilon_{m}}\right)}{\partial t} \varphi d x+\int_{0}^{t} \int_{\Omega}\left(\mathcal{A}\left(x, t, \nabla u^{\varepsilon_{n}}\right)-\mathcal{A}\left(x, t, \nabla u^{\varepsilon_{m}}\right)\right) \nabla \varphi d x d t \\
& +\int_{0}^{t} \int_{\Omega} \lambda\left[\left|u^{\varepsilon_{n}}\right|^{p(x)-2} u^{\varepsilon_{n}}-\left|u^{\varepsilon_{m}}\right|^{p(x)-2} u^{\varepsilon_{m}}\right] \phi d x d s \\
& =\int_{0}^{t} \int_{\Omega}\left[f_{1}^{\varepsilon_{n}}\left(x, t, u^{\varepsilon_{n}}, v^{\varepsilon_{n}}\right)-f_{1}^{\varepsilon_{n}}\left(x, t, u^{\varepsilon_{m}}, v^{\varepsilon_{m}}\right)\right] \varphi d x d s,
\end{aligned}
$$

and

$$
\begin{aligned}
& \int_{\Omega} \frac{\partial b_{\varepsilon_{n}}^{2}\left(v^{\varepsilon_{n}}-v^{\varepsilon_{m}}\right)}{\partial t} \phi d x+\int_{0}^{t} \int_{\Omega}\left(\nabla v^{\varepsilon_{n}}-\nabla v^{\varepsilon_{m}}\right) \nabla \phi d x d t \\
& =\int_{0}^{t} \int_{\Omega}\left[f_{2}^{\varepsilon_{n}}\left(x, t, u^{\varepsilon_{n}}, v^{\varepsilon_{n}}\right)-f_{2}^{\varepsilon_{n}}\left(x, t, u^{\varepsilon_{m}}, v^{\varepsilon_{m}}\right)\right] \phi d x d s
\end{aligned}
$$


where $\varphi \in L^{\infty}(] 0, T\left[; W^{1, p(.)}(\Omega)\right)$ and $\phi \in L^{2}(] 0, T\left[; H_{0}^{1}(\Omega)\right)$. To do this fix $\tau \in[0, T]$. Taking $\varphi=\frac{1}{k} T_{k}\left(u^{\varepsilon_{n}}-u^{\varepsilon_{m}}\right) 1_{\{[0, \tau[\}}$ in (4.48) and $\phi=\frac{1}{k} T_{k}\left(v^{\varepsilon_{n}}-v^{\varepsilon_{m}}\right) 1_{\{[0, \tau[\}}$ in (4.49), one gets

$$
\begin{aligned}
& \frac{1}{k} \int_{\Omega} B_{k}^{1, \varepsilon_{n}}\left(u^{\varepsilon_{n}}(\tau)-u^{\varepsilon_{n}}(\tau)\right) d x-\frac{1}{k} \int_{\Omega} B_{k}^{1, \varepsilon_{n}}\left(u^{\varepsilon_{n}}(0)-u^{\varepsilon_{m}}(0)\right) d x \\
& +\int_{0}^{\tau} \int_{\Omega} \frac{1}{k}\left(\mathcal{A}\left(x, t, \nabla u^{\varepsilon_{n}}\right)-\mathcal{A}\left(x, t, \nabla u^{\varepsilon_{m}}\right)\right) \nabla T_{k}\left(u^{\varepsilon_{n}}-u^{\varepsilon_{m}}\right) d x d t \\
& +\int_{0}^{\tau} \int_{\Omega} \frac{\lambda}{k}\left[\left|u^{\varepsilon_{n}}\right|^{p(x)-2} u^{\varepsilon_{n}}-\left|u^{\varepsilon_{m}}\right|^{p(x)-2} u^{\varepsilon_{m}}\right] T_{k}\left(u^{\varepsilon_{n}}-u^{\varepsilon_{m}}\right) d x d s \\
& =\int_{0}^{t} \int_{\Omega} \frac{1}{k}\left[f_{1}^{\varepsilon_{n}}\left(x, t, u^{\varepsilon_{n}}, v^{\varepsilon_{n}}\right)-f_{1}^{\varepsilon_{n}}\left(x, t, u^{\varepsilon_{m}}, v^{\varepsilon_{m}}\right)\right] T_{k}\left(u^{\varepsilon_{n}}-u^{\varepsilon_{m}}\right) d x d s,
\end{aligned}
$$

and

$$
\begin{aligned}
& \frac{1}{k} \int_{\Omega} B_{k}^{2, \varepsilon_{n}}\left(v^{\varepsilon_{n}}(\tau)-v^{\varepsilon_{m}}(\tau)\right) d x-\frac{1}{k} \int_{\Omega} B_{k}^{2, \varepsilon_{n}}\left(v^{\varepsilon_{n}}(0)-v^{\varepsilon_{m}}(0)\right) d x \\
& +\frac{1}{k} \int_{0}^{t} \int_{\Omega} \nabla\left(v^{\varepsilon_{n}}-v^{\varepsilon_{m}}\right) \nabla T_{k}\left(v^{\varepsilon_{n}}-v^{\varepsilon_{m}}\right) d x d t \\
& =\int_{0}^{t} \int_{\Omega} \frac{1}{k}\left[f_{2}^{\varepsilon_{n}}\left(x, t, u^{\varepsilon_{n}}, v^{\varepsilon_{n}}\right)-f_{2}^{\varepsilon_{n}}\left(x, t, u^{\varepsilon_{m}}, v^{\varepsilon_{m}}\right)\right] T_{k}\left(v^{\varepsilon_{n}}-v^{\varepsilon_{m}}\right) d x d s
\end{aligned}
$$

where

$$
B_{k}^{i, \varepsilon_{n}}(r)=\int_{0}^{r} T_{k}(s) \frac{\partial b_{\varepsilon_{n}}^{i}(s)}{\partial s} d s . \forall i=\overline{1,2}
$$

adding (4.50) and (4.51), we get

$$
\begin{aligned}
& \frac{1}{k} \int_{\Omega} B_{k}^{1, \varepsilon_{n}}\left(u^{\varepsilon_{n}}(\tau)-u^{\varepsilon_{m}}(\tau)\right) d x+\frac{1}{k} \int_{\Omega} B_{k}^{2, \varepsilon_{n}}\left(v^{\varepsilon_{n}}(\tau)-v^{\varepsilon_{m}}(\tau)\right) d x \\
& \leq \int_{0}^{\tau} \int_{\Omega} \lambda\left[\left|u^{\varepsilon_{n}}\right|^{p(x)-2} u^{\varepsilon_{n}}-\left|u^{\varepsilon_{m}}\right|^{p(x)-2} u^{\varepsilon_{m}}\right] d x d t+ \\
& \int_{0}^{\tau} \int_{\Omega}\left[f_{1}^{\varepsilon_{n}}\left(x, t, u^{\varepsilon_{n}}, v^{\varepsilon_{n}}\right)-f_{1}^{\varepsilon_{n}}\left(x, t, u^{\varepsilon_{m}}, v^{\varepsilon_{m}}\right)\right] d x d t+ \\
& \int_{0}^{\tau} \int_{\Omega}\left[f_{2}^{\varepsilon_{n}}\left(x, t, u^{\varepsilon_{n}}, v^{\varepsilon_{n}}\right)-f_{2}^{\varepsilon_{n}}\left(x, t, u^{\varepsilon_{m}}, v^{\varepsilon_{m}}\right)\right] d x d t+ \\
& \int_{\Omega}\left|b_{\varepsilon_{n}}^{1}\left(u_{0}^{\varepsilon_{n}}-u_{0}^{\varepsilon_{m}}\right)\right| d x+\int_{\Omega}\left|b_{\varepsilon_{n}}^{2}\left(v_{0}^{\varepsilon_{n}}-v_{0}^{\varepsilon_{m}}\right)\right| d x
\end{aligned}
$$


since $B_{k}^{i, \varepsilon_{n}}(r) \geq \rho \int_{0}^{r} T_{k}(s) d s \geq \rho(|s|-1) \cdot \forall i=\overline{1,2}$

$$
\begin{aligned}
& \int_{\Omega}\left|u^{\varepsilon_{n}}(\tau)-u^{\varepsilon_{m}}(\tau)\right| d x+\int_{\Omega}\left|v^{\varepsilon_{n}}(\tau)-v^{\varepsilon_{m}}(\tau)\right| d x \\
& \leq 2 k \operatorname{meas}(\Omega)+\int_{0}^{\tau} \int_{\Omega} k \lambda\left[\left|u^{\varepsilon_{n}}\right|^{p(x)-2} u^{\varepsilon_{n}}-\left|u^{\varepsilon_{m}}\right|^{p(x)-2} u^{\varepsilon_{m}}\right] d x d t \\
& +k \int_{0}^{\tau} \int_{\Omega}\left[f_{1}^{\varepsilon_{n}}\left(x, t, u^{\varepsilon_{n}}, v^{\varepsilon_{n}}\right)-f_{1}^{\varepsilon_{n}}\left(x, t, u^{\varepsilon_{m}}, v^{\varepsilon_{m}}\right)\right] d x d t \\
& +k \int_{0}^{\tau} \int_{\Omega}\left[f_{2}^{\varepsilon_{n}}\left(x, t, u^{\varepsilon_{n}}, v^{\varepsilon_{n}}\right)-f_{2}^{\varepsilon_{n}}\left(x, t, u^{\varepsilon_{m}}, v^{\varepsilon_{m}}\right)\right] d x d t \\
& +k \int_{\Omega}\left|b_{\varepsilon_{n}}^{1}\left(u_{0}^{\varepsilon_{n}}-u_{0}^{\varepsilon_{m}}\right)\right| d x+k \int_{\Omega}\left|b_{\varepsilon_{n}}^{2}\left(v_{0}^{\varepsilon_{n}}-v_{0}^{\varepsilon_{m}}\right)\right| d x,
\end{aligned}
$$

letting $\varepsilon_{n}, \varepsilon_{m} \rightarrow \infty$ and them $k \rightarrow 0$, to obtain

$$
\begin{aligned}
& \sup _{\tau \in[0, T]} \int_{\Omega}\left|u^{\varepsilon_{n}}(\tau)-u^{\varepsilon_{m}}(\tau)\right| d x+\sup _{\tau \in[0, T]} \int_{\Omega}\left|v^{\varepsilon_{n}}(\tau)-v^{\varepsilon_{m}}(\tau)\right| d x \\
& \leq \int_{0}^{\tau} \int_{\Omega} k \lambda\left[\left|u^{\varepsilon_{n}}\right|^{p(x)-2} u^{\varepsilon_{n}}-\left|u^{\varepsilon_{m}}\right|^{p(x)-2} u^{\varepsilon_{m}}\right] d x d t \\
& +k \int_{0}^{\tau} \int_{\Omega}\left[f_{1}^{\varepsilon_{n}}\left(x, t, u^{\varepsilon_{n}}, v^{\varepsilon_{n}}\right)-f_{1}^{\varepsilon_{n}}\left(x, t, u^{\varepsilon_{m}}, v^{\varepsilon_{m}}\right)\right] d x d t \\
& +k \int_{0}^{\tau} \int_{\Omega}\left[f_{2}^{\varepsilon_{n}}\left(x, t, u^{\varepsilon_{n}}, v^{\varepsilon_{n}}\right)-f_{2}^{\varepsilon_{n}}\left(x, t, u^{\varepsilon_{m}}, v^{\varepsilon_{m}}\right)\right] d x d t \\
& +k \int_{\Omega}\left|b_{\varepsilon_{n}}^{1}\left(u_{0}^{\varepsilon_{n}}-u_{0}^{\varepsilon_{m}}\right)\right| d x+k \int_{\Omega}\left|b_{\varepsilon_{n}}^{2}\left(v_{0}^{\varepsilon_{n}}-v_{0}^{\varepsilon_{m}}\right)\right| d x .
\end{aligned}
$$

- Step 2: The limit of the solution of the approximated problem. Arguing again as in [ [3], [4], [5]] estimates (4.38), (4.40), (4.39) and (4.41) imply that, for a subsequence still indexed by $\varepsilon$,

$$
\left(u^{\varepsilon}, v^{\varepsilon}\right) \text { converge almost every where to }(u, v) \text {, }
$$

using (4.15), (4.34), (4.35) and (4.44), to get

$$
T_{k}\left(u^{\varepsilon}\right) \text { converge weakly to } T_{k}(u) \text { in } L^{p-}(] 0, T\left[; W_{0}^{1, p(\cdot)}(\Omega)\right)
$$

and

$$
T_{k}\left(v^{\varepsilon}\right) \text { converge weakly to } T_{k}(v) \text { in } L^{2}(] 0, T\left[; H_{0}^{1}(\Omega)\right) \text {, }
$$




$$
\chi_{\left\{\left|u^{\varepsilon}\right| \leq k\right\}} \mathcal{A}\left(x, t, \nabla u^{\varepsilon}\right) \rightarrow \eta_{k} \text { weakly in }\left(L^{p^{\prime}(.)}(Q)\right)^{N},
$$

as $\varepsilon$ tends to 0 for any $k>0$ and any $n \geq 1$ and where for any $k>0, \eta_{k}$ belongs to $\left(L^{p^{\prime}(\cdot)}(Q)\right)^{N}$. Since $\gamma\left(u^{\varepsilon}\right)$ is a continuous incrassing function, from the monotone convergence theorem and (4.25) and by (4.52), to obtain that

$$
\gamma\left(u^{\varepsilon}\right) \text { converge weakly to } \gamma(u) \text { in } L^{1}(Q) \text {. }
$$

We now establish that $\left(b_{1}(u), b_{2}(v)\right)$ belongs to $\left(L^{\infty}(] 0, T\left[; L^{1}(\Omega)\right)\right)^{2}$. Indeed using (4.20) and $\left|B_{k}^{i, \varepsilon}(s)\right| \geq \rho(|s|-1), \forall i=\overline{1,2}$, leads to

$$
\begin{aligned}
\int_{\Omega}\left|b_{\varepsilon}^{1}\left(u^{\varepsilon}\right)\right|(t) d x+\int_{\Omega}\left|b_{\varepsilon}^{2}\left(v^{\varepsilon}\right)\right|(t) d x & \leq \rho(2 \operatorname{meas}(\Omega) \\
& +\left\|\left(f_{1}^{\varepsilon}\left(x, t, u^{\varepsilon}, v^{\varepsilon}\right), f_{2}^{\varepsilon}\left(x, t, u^{\varepsilon}, v^{\varepsilon}\right)\right)\right\|_{\left(L^{1}(Q)\right)^{2}} \\
& +k\left\|\gamma\left(u^{\varepsilon}\right)\right\|_{L^{1}(Q)} \\
& \left.+k\left\|\left(b_{\varepsilon}^{1}\left(u_{0}^{\varepsilon}\right), b_{\varepsilon}^{2}\left(v_{0}^{\varepsilon}\right)\right)\right\|_{\left(L^{1}(\Omega)\right)^{2}}\right) .
\end{aligned}
$$

By lemma (4.1) and (4.46), (4.47), we conclude that there exist two subsequences of $u^{\varepsilon_{n}}$ and $v^{\varepsilon_{n}}$, still denoted by themselves for convenience, such that $u^{\varepsilon_{n}}$ converges to a function $u$ in $C(] 0, T\left[; L^{1}(\Omega)\right)$, $v^{\varepsilon_{n}}$ converges to a function $v$ in $C(] 0, T\left[; L^{1}(\Omega)\right)$. Using (4.25) and (4.10), (4.26), we have $\left(b_{1}(u), b_{2}(v)\right)$ belongs to $\left(L^{\infty}(] 0, T\left[; L^{1}(\Omega)\right)\right)^{2}$. We are now in a position to exploit $(4.46)$ and (4.47). Since $\left(u^{\varepsilon}, v^{\varepsilon}\right)$ is bounded in $\left(L^{\infty}(] 0, T\left[; L^{1}(\Omega)\right)\right)^{2}$, to get

$$
\lim _{n \rightarrow+\infty}\left(\operatorname{supmeas}_{\varepsilon}\left\{\left|u^{\varepsilon}\right| \geq n\right\}\right)=0 \text {. }
$$

and

$$
\lim _{n \rightarrow+\infty}\left(\operatorname{supmeas}_{\varepsilon}\left\{\left|v^{\varepsilon}\right| \geq n\right\}\right)=0 .
$$

The equi-integrability of the sequence $f_{i}^{\varepsilon}\left(x, t, u^{\varepsilon}, v^{\varepsilon}\right)$ in $\left(L^{1}(Q)\right)^{2}$. We shall now prove that $f_{i}^{\varepsilon}\left(x, t, u^{\varepsilon}, v^{\varepsilon}\right)$ converges to $f_{i}(x, t, u, v)$ strongly in $\left(L^{1}(Q)\right)^{2}$, for all $i=\overline{1,2}$ by using Vitali's theorem. Since $f_{i}^{\varepsilon}\left(x, t, u^{\varepsilon}, v^{\varepsilon}\right) \rightarrow f_{i}(x, t, u, v)$ a.e in $Q$ it suffices to prove that $f_{i}^{\varepsilon}\left(x, t, u^{\varepsilon}, v^{\varepsilon}\right)$ are equi-integrable in $Q$. Let $\delta_{1}>0$ and $\mathbf{A}$ be a measurable subset belonging to $\left.\Omega \times\right] 0, T$, we define the following sets

$$
\begin{aligned}
& G_{\delta_{1}}=\left\{(x, t) \in Q:\left|u_{n}\right| \leq \delta_{1}\right\} \\
& F_{\delta_{1}}=\left\{(x, t) \in Q:\left|u_{n}\right|>\delta_{1}\right\} .
\end{aligned}
$$

Using the generalized Hölder's inequality and Poincaré inequality, to have

$$
\int_{\mathbf{A}}\left|f_{1}^{\varepsilon}\left(x, t, u^{\varepsilon}, v^{\varepsilon}\right)\right| d x d t=\int_{\mathbf{A} \cap G_{\delta_{1}}}\left|f_{1}^{\varepsilon}\left(x, t, u^{\varepsilon}, v^{\varepsilon}\right)\right| d x d t+\int_{\mathbf{A} \cap F_{\delta_{1}}}\left|f_{1}^{\varepsilon}\left(x, t, u^{\varepsilon}, v^{\varepsilon}\right)\right| d x d t,
$$


therfore

$$
\begin{aligned}
& \int_{\mathbf{A}}\left|f_{1}^{\varepsilon}\left(x, t, u^{\varepsilon}, v^{\varepsilon}\right)\right| d x d t \leq \int_{\mathbf{A} \cap G_{\delta_{1}}}\left(c_{k, \varepsilon}(x, t)+\sigma_{k, \varepsilon}\left|v^{\varepsilon}\right|^{2}\right) d x d t \\
& +\int_{\mathbf{A} \cap F_{\delta_{1}}}\left|f_{1}^{\varepsilon}\left(x, t, u^{\varepsilon}, v^{\varepsilon}\right)\right| d x d t \\
& \leq \int_{\mathbf{A}} c_{k, \varepsilon}(x, t) d x d t+\sigma_{k, \varepsilon} \int_{Q}\left|\nabla T_{\delta_{1}}\left(v^{\varepsilon}\right)\right|^{2} d x d t \\
& +\int_{\mathbf{A} \cap F_{\delta_{1}}}\left|f_{1}^{\varepsilon}\left(x, t, u^{\varepsilon}, v^{\varepsilon}\right)\right| d x d t \\
& \leq \int_{\mathbf{A}} c_{k, \varepsilon}(x, t) d x d t+\sigma_{k, \varepsilon}(\operatorname{meas}(\mathbf{Q})+1)^{\frac{1}{2}} \\
& \left(\int_{Q_{T}}\left|\nabla T_{\delta_{1}}\left(v^{\varepsilon}\right)\right|^{2} d x d t\right)^{\frac{1}{2}}+\int_{\mathbf{A} \cap F_{\delta_{1}}}\left|f_{1}^{\varepsilon}\left(x, t, u^{\varepsilon}, v^{\varepsilon}\right)\right| d x d t \\
& \leq K_{1}+C_{2}\left(\frac{k}{\alpha}\left\|b_{\varepsilon}^{2}\left(v_{0}^{\varepsilon}\right)\right\|_{L^{1}(\Omega)}\right)^{\frac{1}{2}} \\
& +\int_{\mathbf{A} \cap F_{\delta_{1}}} \frac{1}{\left|u^{\varepsilon}\right|}\left|u^{\varepsilon} f_{1}^{\varepsilon}\left(x, t, u^{\varepsilon}, v^{\varepsilon}\right)\right| d x d t \\
& \leq K_{2}+\int_{\mathbf{A} \cap F_{\delta_{1}}} \frac{1}{\delta_{1}}\left|u^{\varepsilon} f_{1}^{\varepsilon}\left(x, t, u^{\varepsilon}, v^{\varepsilon}\right)\right| d x d t \\
& \leq K_{2}+\frac{1}{\delta_{1}}\left(\frac{1}{p^{-}}+\frac{1}{p^{-}}\right)\left(\int_{\mathbf{A} \cap F_{\delta_{1}}}\left|u^{\varepsilon}\right|^{p(x)} d x d t\right)^{\frac{1}{p^{-}}} \\
& \left(\int_{\mathbf{A} \cap F_{\delta_{1}}}\left|f_{1}^{\varepsilon}\left(x, t, u^{\varepsilon}, v^{\varepsilon}\right)\right|^{p^{\prime}(x)(p(x)-1)} d x d t\right)^{\frac{1}{p^{\prime-}}} \\
& \rightarrow \quad 0 \text { when meas }(\mathbf{A}) \rightarrow \mathbf{0} \text {. }
\end{aligned}
$$

Which shows that $f_{1}^{\varepsilon}\left(x, t, u^{\varepsilon}, v^{\varepsilon}\right)$ is equi-integrable. By using Vitali's theorem, to get

$$
f_{1}^{\varepsilon}\left(x, t, u^{\varepsilon}, v^{\varepsilon}\right) \rightarrow f_{1}(x, t, u, v) \text { strongly in } L^{1}(Q) .
$$

Now we prove that

$$
f_{2}^{\varepsilon}\left(x, t, u^{\varepsilon}, v^{\varepsilon}\right) \rightarrow f_{2}(x, t, u, v) \text { strongly in } L^{1}(Q) .
$$


Let $\delta_{2}>0$ and $\mathbf{A}$ be a measurable subset belonging to $\left.\Omega \times\right] 0, T$, we define the following sets

$$
\begin{aligned}
& G_{\delta_{2}}=\left\{(x, t) \in Q:\left|v_{n}\right| \leq \delta_{2}\right\}, \\
& F_{\delta_{2}}=\left\{(x, t) \in Q:\left|v_{n}\right|>\delta_{2}\right\} .
\end{aligned}
$$

Using the generalized Hölder's inequality and Poincaré inequality, to get

$$
\int_{\mathbf{A}}\left|f_{2}^{\varepsilon}\left(x, t, u^{\varepsilon}, v^{\varepsilon}\right)\right| d x d t=\int_{\mathbf{A} \cap G_{\delta_{2}}}\left|f_{2}^{\varepsilon}\left(x, t, u^{\varepsilon}, v^{\varepsilon}\right)\right| d x d t+\int_{\mathbf{A} \cap F_{\delta_{2}}}\left|f_{2}^{\varepsilon}\left(x, t, u^{\varepsilon}, v^{\varepsilon}\right)\right| d x d t,
$$

therfore

$$
\begin{aligned}
& \int_{\mathbf{A}}\left|f_{2}^{\varepsilon}\left(x, t, u^{\varepsilon}, v^{\varepsilon}\right)\right| d x d t \leq \int_{\mathbf{A} \cap G_{\delta_{2}}}\left(G_{k}^{\varepsilon}(x, t)+\xi_{k}^{\varepsilon}\left|u^{\varepsilon}\right|^{p(x)-1}\right) d x d t \\
& +\int_{\mathbf{A} \cap F_{\delta_{2}}}\left|f_{2}^{\varepsilon}\left(x, t, u^{\varepsilon}, v^{\varepsilon}\right)\right| d x d t \\
& \leq \int_{\mathbf{A}} G_{k}^{\varepsilon}(x, t) d x d t+\xi_{k}^{\varepsilon} \int_{Q}\left|\nabla T_{\delta_{2}}\left(u^{\varepsilon}\right)\right|^{p(x)-1} d x d t \\
& +\int_{\mathbf{A} \cap F_{\delta_{2}}}\left|f_{2}^{\varepsilon}\left(x, t, u^{\varepsilon}, v^{\varepsilon}\right)\right| d x d t \\
& \leq \int_{\mathbf{A}} G_{k}^{\varepsilon}(x, t) d x d t+\xi_{k}^{\varepsilon}\left(\frac{1}{p^{-}}+\frac{1}{p^{\prime-}}\right)(\operatorname{meas}(\mathbf{Q})+1)^{\frac{1}{p^{-}}} \\
& \left(\int_{Q_{T}}\left|\nabla T_{\delta_{2}}\left(u^{\varepsilon}\right)\right|^{(p(x)-1) p^{\prime}(x)} d x d t\right)^{\frac{1}{p^{\prime-}}} \\
& +\int_{\mathbf{A} \cap F_{\delta_{2}}}\left|f_{2}^{\varepsilon}\left(x, t, u^{\varepsilon}, v^{\varepsilon}\right)\right| d x d t \\
& \leq K_{3}+C_{4}\left(\frac{k}{\alpha}\left\|b_{\varepsilon}^{1}\left(u_{0}^{\varepsilon}\right)\right\|_{L^{1}(\Omega)}\right)^{\frac{1}{2}} \\
& +\int_{\mathbf{A} \cap F_{\delta_{2}}} \frac{1}{\left|v^{\varepsilon}\right|}\left|v^{\varepsilon} f_{2}^{\varepsilon}\left(x, t, u^{\varepsilon}, v^{\varepsilon}\right)\right| d x d t \\
& \leq K_{4}+\int_{\mathbf{A} \cap F_{\delta_{2}}} \frac{1}{\delta_{2}}\left|v^{\varepsilon} f_{2}^{\varepsilon}\left(x, t, u^{\varepsilon}, v^{\varepsilon}\right)\right| d x d t \\
& \leq K_{4}+\frac{1}{\delta_{2}}\left(\int_{\mathbf{A} \cap F_{\delta_{2}}}\left|v^{\varepsilon}\right|^{2} d x d t\right)^{\frac{1}{2}}\left(\int_{\mathbf{A} \cap F_{\delta_{2}}}\left|f_{2}^{\varepsilon}\left(x, t, u^{\varepsilon}, v^{\varepsilon}\right)\right|^{2} d x d t\right)^{\frac{1}{2}} \\
& \rightarrow 0 \text { when meas }(\mathbf{A}) \rightarrow \mathbf{0} \text {. }
\end{aligned}
$$

Which shows that $f_{2}^{\varepsilon}\left(x, t, u^{\varepsilon}, v^{\varepsilon}\right)$ is equi-integrable. By using Vitali's theorem, to get

$$
f_{2}^{\varepsilon}\left(x, t, u^{\varepsilon}, v^{\varepsilon}\right) \rightarrow f_{2}(x, t, u, v) \text { strongly in } L^{1}(Q) .
$$


Using (4.56), (4.61) and the equi-integrability of the sequence $\left|b_{\varepsilon}^{1}\left(u_{0}^{\varepsilon}\right)\right|$ in $L^{1}(\Omega)$ and $\left|b_{\varepsilon}^{2}\left(v_{0}^{\varepsilon}\right)\right|$ in $L^{1}(\Omega)$, we deduce that

$$
\lim _{n \rightarrow+\infty}\left(\sup _{\varepsilon}\left(\int_{\left\{n \leq\left|u^{\varepsilon}\right| \leq n+1\right\}} \mathcal{A}\left(x, t, \nabla u^{\varepsilon}\right) \nabla u^{\varepsilon} d x d t+\int_{\left\{n \leq\left|v^{\varepsilon}\right| \leq n+1\right\}}\left|\nabla v^{\varepsilon}\right|^{2} d x d t\right)\right)=0 .
$$

- Step 4: Strong convergence. The specifie time regularization of $T_{k}(u)$ (for fixed $k \geq 0$ ) is defined as follows. Let $\left(v_{0}^{\mu}\right)_{\mu}$ be a sequaence in $L^{\infty}(\Omega) \cap W_{0}^{1, p(.)}(\Omega)$ such that $\left\|v_{0}^{\mu}\right\|_{L^{\infty}(\Omega)} \leq k, \forall \mu>0$, and $v_{0}^{\mu} \rightarrow T_{k}\left(u_{0}\right)$ a.e in $\Omega$ with $\frac{1}{\mu}\left\|v_{0}^{\mu}\right\|_{L^{p(.)}(\Omega)} \rightarrow 0$ as $\mu \rightarrow+\infty$.

For fixed $k \geq 0$ and $\mu>0$, let us consider the unique solution $T_{k}(u)_{\mu} \in L^{\infty}(\Omega) \cap$ $L^{p-}(] 0, T\left[; W_{0}^{1, p(.)}(\Omega)\right)$ of the monotone problem

$$
\begin{gathered}
\frac{\partial T_{k}(u)_{\mu}}{\partial t}+\mu\left(T_{k}(u)_{\mu}-T_{k}(u)\right)=0 \text { in } \mathcal{D}^{\prime}(Q), \\
T_{k}(u)_{\mu}(t=0)=v_{0}^{\mu}
\end{gathered}
$$

The behavior of $T_{k}(u)_{\mu}$ as $\mu \rightarrow+\infty$ is investigated in [9] and we just recall here that (4.67)-(4.68) imply that

$$
T_{k}(u)_{\mu} \rightarrow T_{k}(u) \text { strongly in } L^{p-}(] 0, T\left[; W_{0}^{1, p(.)}(\Omega)\right) \text { a.e in } Q, \text { as } \mu \rightarrow+\infty
$$

with $\left\|T_{k}(u)_{\mu}\right\|_{L^{\infty}(\Omega)} \leq k$, for any $\mu$, and $\frac{\partial T_{k}(u)_{\mu}}{\partial t} \in L^{(p-)^{\prime}}(] 0, T\left[; W^{-1, p^{\prime}(.)}(\Omega)\right)$.

The main estimate is the following

Lemma 4.2. Let $S$ be an increasing $C^{\infty}(\mathbb{R})-$ function such that $S(r)=r$ for $r \leq k$, and supp $S^{\prime}$ is compact. Then

$$
\liminf _{\mu \rightarrow+\infty} \lim _{\varepsilon \rightarrow 0} \int_{0}^{T}\left\langle\frac{\partial B_{S}^{1}\left(u^{\varepsilon}\right)}{\partial t},\left(T_{k}\left(u^{\varepsilon}\right)_{\mu}-T_{k}(u)\right)\right\rangle d t \geq 0,
$$

where here $\langle.,$.$\rangle denotes the duality pairing between L^{1}(\Omega)+W^{-1, p^{\prime}(.)}(\Omega)$ and $L^{\infty}(\Omega) \cap W_{0}^{1, p(.)}(\Omega)$, and where $B_{S}^{1}(z)=\int_{0}^{z} b_{1}^{\prime}(r) S^{\prime}(r) d r$.

Proof. See [5], Lemma 1.

Now we are to prove that the weak limit $\eta_{k}$ and we prove the weak $L^{1}$ convergence of the "truncted" energy $\mathcal{A}\left(x, t, \nabla T_{k}\left(u^{\varepsilon}\right)\right)$ as $\varepsilon$ tends to 0 . In order to show this result we recall the lemma below.

Lemma 4.3. The subsequence of $u^{\varepsilon}$ defined in step 3 satisfies

$$
\limsup _{\varepsilon \rightarrow 0} \int_{Q} \mathcal{A}\left(x, t, \nabla u^{\varepsilon}\right) \nabla T_{k}\left(u^{\varepsilon}\right) d x d t \leq \int_{Q} \eta_{k} \nabla T_{k}(u) d x d t
$$




$$
\begin{array}{r}
\lim _{\varepsilon \rightarrow 0} \int_{Q}\left[\mathcal{A}\left(x, t, \nabla u_{\chi_{\left\{\left|u^{\varepsilon}\right| \leq k\right\}}^{\varepsilon}}^{\varepsilon}\right)-\mathcal{A}\left(x, t, \nabla u_{\chi_{\{|u| \leq k\}}}\right)\right] \\
\times\left[\nabla u_{\chi_{\left\{\left|u^{\varepsilon}\right| \leq k\right\}}^{\varepsilon}}^{\varepsilon}-\nabla u_{\chi_{\{|| \leq k\}}}\right] d x d t=0
\end{array}
$$

$\eta_{k}=\mathcal{A}\left(x, t, \nabla u_{\chi_{\{|u| \leq k\}}}\right)$ a.e in $Q$, for any $k \geq 0$, as $\varepsilon$ tends to 0 .

$$
\mathcal{A}\left(x, t, \nabla u^{\varepsilon}\right) \nabla T_{k}\left(u^{\varepsilon}\right) \rightarrow \mathcal{A}(x, t, \nabla u) \nabla T_{k}(u) \text { weakly in } L^{1}(Q) .
$$

Proof. Let us introduce a sequence of increasing $C^{\infty}(\mathbb{R})$-functions $S_{n}$ such that, for any $n \geq 1$

$$
\left\{\begin{array}{c}
S_{n}(r)=r \text { if }|\mathrm{r}| \leq n, \\
\operatorname{supp}\left(S_{n}^{\prime}\right) \subset[-(n+1),(n+1)], \\
\left\|S_{n}^{\prime \prime}\right\|_{L^{\infty}(\mathbb{R})} \leq 1 .
\end{array}\right.
$$

For fixed $k \geq 0$, we consider the test function $S_{n}^{\prime}\left(u^{\varepsilon}\right)\left(T_{k}\left(u_{\varepsilon}\right)-\left(T_{k}(u)\right)_{\mu}\right)$ in (4.15), we use the definition (4.73) of $S_{n}^{\prime}$ and we definie $W_{\mu}^{\varepsilon}=T_{k}\left(u_{\varepsilon}\right)-\left(T_{k}(u)\right)_{\mu}$, to get

$$
\begin{aligned}
& \int_{0}^{T}\left\langle\left(B_{S}^{1}\left(u^{\varepsilon}\right)\right)_{t}, W_{\mu}^{\varepsilon}\right\rangle d t+\int_{Q} S_{n}^{\prime}\left(u^{\varepsilon}\right) \mathcal{A}\left(x, t, \nabla u^{\varepsilon}\right) \nabla W_{\mu}^{\varepsilon} d x d t \\
& +\int_{Q} S_{n}^{\prime \prime}\left(u^{\varepsilon}\right) \mathcal{A}\left(x, t, \nabla u^{\varepsilon}\right) \nabla u^{\varepsilon} W_{\mu}^{\varepsilon} d x d t+\int_{Q} \gamma\left(u^{\varepsilon}\right) S_{n}^{\prime}\left(u^{\varepsilon}\right) W_{\mu}^{\varepsilon} d x d t \\
= & \int_{Q} f_{1}^{\varepsilon}\left(x, t, u^{\varepsilon}, v^{\varepsilon}\right) S_{n}^{\prime}\left(u^{\varepsilon}\right) W_{\mu}^{\varepsilon} d x d t .
\end{aligned}
$$

Now we pass to the limit in (4.74) as $\varepsilon \rightarrow 0, \mu \rightarrow+\infty, n \rightarrow+\infty$ for $k$ real number fixed. In order to perform this task, we prove below the following results for any $k \geq 0$ :

$$
\begin{gathered}
\liminf _{\mu \rightarrow+\infty \varepsilon \rightarrow 0} \lim _{0}^{T}\left\langle\left(B_{S}^{1}\left(u^{\varepsilon}\right)\right)_{t}, W_{\mu}^{\varepsilon}\right\rangle d t \geq 0 \text { for any } n \geq k, \\
\lim _{n \rightarrow+\infty \mu \rightarrow+\infty \varepsilon \rightarrow 0} \lim _{Q} \lim _{n} S_{n}^{\prime \prime}\left(u^{\varepsilon}\right) \mathcal{A}\left(x, t, \nabla u^{\varepsilon}\right) \nabla u^{\varepsilon} W_{\mu}^{\varepsilon} d x d t=0, \\
\lim _{\mu \rightarrow+\infty \varepsilon \rightarrow 0} \lim _{Q} \gamma\left(u^{\varepsilon}\right) S_{n}^{\prime}\left(u^{\varepsilon}\right) W_{\mu}^{\varepsilon} d x d t=0, \text { for any } n \geq 1, \\
\lim _{\mu \rightarrow+\infty \varepsilon \rightarrow 0} \lim _{Q} f_{1}^{\varepsilon}\left(x, t, u^{\varepsilon}, v^{\varepsilon}\right) S_{n}^{\prime}\left(u^{\varepsilon}\right) W_{\mu}^{\varepsilon} d x d t=0, \text { for any } n \geq 1 .
\end{gathered}
$$

Proof of (4.75). In view of the definition $W_{\mu}^{\varepsilon}$, we apply lemma (4.2) with $S=S_{n}$ for fixed $n \geq k$. As a consequence, (4.75) hold true. 
Proof of (4.76). For any $n \geq 1$ fixed, we have $\operatorname{supp}\left(S_{n}^{\prime \prime}\right) \subset[-(n+1),-n] \cup[n, n+1],\left\|W_{\mu}^{\varepsilon}\right\|_{L^{\infty}(Q)} \leq$ $2 k$ and $\left\|S_{n}^{\prime \prime}\right\|_{L^{\infty}(\mathbb{R})} \leq 1$, to get

$$
\begin{array}{r}
\left|\int_{Q} S_{n}^{\prime \prime}\left(u^{\varepsilon}\right) \mathcal{A}\left(x, t, \nabla u^{\varepsilon}\right) \nabla u^{\varepsilon} W_{\mu}^{\varepsilon} d x d t\right| \\
\leq 2 k \int_{\left\{n \leq\left|u^{\varepsilon}\right| \leq n+1\right\}} \mathcal{A}\left(x, t, \nabla u^{\varepsilon}\right) \nabla u^{\varepsilon} d x d t,
\end{array}
$$

for any $n \geq 1$, by (4.66) it possible to etablish (4.76)

Proof of (4.77). For fixed $n \geq 1$ and in view (4.56) . Lebesgue's convergence theorem implies that for any $\mu>0$ and any $n \geq 1$

$$
\lim _{\varepsilon \rightarrow 0} \int_{Q} \gamma\left(u^{\varepsilon}\right) S_{n}^{\prime}\left(u^{\varepsilon}\right) W_{\mu}^{\varepsilon} d x d t=\int_{Q} \gamma(u) S_{n}^{\prime}(u)\left(T_{k}(u)-T_{k}(u)_{\mu}\right) d x d t .
$$

Appealing now to (4.69) and passing to the limit as $\mu \rightarrow+\infty$ in (4.80) allows to conclude that (4.77) holds true.

Proof of (4.78). By (4.11), (4.61) and Lebesgue's convergence theorem implies that for any $\mu>0$ and any $n \geq 1$, it is possible to pass to the limit for $\varepsilon \rightarrow 0$

$$
\lim _{\varepsilon \rightarrow 0} \int_{Q} f_{1}^{\varepsilon}\left(x, t, u^{\varepsilon}, v^{\varepsilon}\right) S_{n}^{\prime}\left(u^{\varepsilon}\right) W_{\mu}^{\varepsilon} d x d t=\int_{Q} f_{1}(x, t, u, v) S_{n}^{\prime}(u)\left(T_{k}(u)-T_{k}(u)_{\mu}\right) d x d t,
$$

using (4.69) permits to the limit as $\mu$ tends to $+\infty$ in the above equality to obtain (4.78).

Now turn back to the proof of Lemma (4.3), due to (4.75)-(4.78), we are in a position to pass to the limit-sup when $\varepsilon \rightarrow 0$, then to the limit-sup when $\mu \rightarrow+\infty$ and then to the limit as $n \rightarrow+\infty$ in (4.74). Using the definition of $W_{\mu}^{\varepsilon}$, we deduce that for any $k \geq 0$,

$$
\lim _{n \rightarrow+\infty} \operatorname{limsuplimsup}_{\mu \rightarrow+\infty} \int_{\varepsilon \rightarrow 0} \mathcal{A}\left(x, t, \nabla u^{\varepsilon}\right) S_{n}^{\prime}\left(u^{\varepsilon}\right) \nabla\left(T_{k}\left(u^{\varepsilon}\right)-T_{k}(u)_{\mu}\right) d x d t \leq 0 .
$$

Since $\mathcal{A}\left(x, t, \nabla u^{\varepsilon}\right) S_{n}^{\prime}\left(u^{\varepsilon}\right) \nabla T_{k}\left(u^{\varepsilon}\right)=\mathcal{A}\left(x, t, \nabla u^{\varepsilon}\right) \nabla T_{k}\left(u^{\varepsilon}\right)$ fo $k \leq n$, the above inequality implies that for $k \leq n$,

$$
\begin{aligned}
& \limsup _{\varepsilon \rightarrow 0} \int_{Q} \mathcal{A}\left(x, t, \nabla u^{\varepsilon}\right) \nabla T_{k}\left(u^{\varepsilon}\right) d x d t \\
\leq & \lim _{n \rightarrow+\infty} \operatorname{limsuplimsup}_{\mu \rightarrow+\infty} \int_{\varepsilon \rightarrow 0} \mathcal{A}\left(t, x, \nabla u^{\varepsilon}\right) S_{n}^{\prime}\left(u^{\varepsilon}\right) \nabla T_{k}(u)_{\mu} d x d t .
\end{aligned}
$$

Due to (4.55), to have

$$
\mathcal{A}\left(x, t, \nabla u^{\varepsilon}\right) S_{n}^{\prime}\left(u^{\varepsilon}\right) \rightarrow \eta_{n+1} S_{n}^{\prime}(u) \text { weakly in }\left(L^{p^{\prime}(.)}(Q)\right)^{N} \text { as } \varepsilon \rightarrow 0,
$$


and the strong convergence of $T_{k}(u)_{\mu}$ to $T_{k}(u)$ in $L^{p^{-}}(] 0, T\left[; W_{0}^{1, p}(\Omega)\right)$ as $\mu \rightarrow+\infty$, to get

$$
\begin{gathered}
\lim _{u \rightarrow+\infty \varepsilon \rightarrow 0} \lim _{Q} \mathcal{A}\left(x, t, \nabla u^{\varepsilon}\right) S_{n}^{\prime}\left(u^{\varepsilon}\right) \nabla T_{k}(u)_{\mu} d x d t \\
=\int_{Q} S_{n}^{\prime}(u) \eta_{n+1} \nabla T_{k}(u) d x d t=\int_{Q} \eta_{n+1} \nabla T_{k}(u) d x d t
\end{gathered}
$$

as soon as $k \leq n$, since $S_{n}^{\prime}(s)=1$ for $|s| \leq n$. Now, for $k \leq n$, to have

$$
S_{n}^{\prime}\left(u^{\varepsilon}\right) \mathcal{A}\left(x, t, \nabla u^{\varepsilon}\right)_{\chi_{\left\{\left|u^{\varepsilon}\right| \leq k\right\}}}=\mathcal{A}\left(x, t, \nabla u^{\varepsilon}\right)_{\chi_{\left\{\left|u^{\varepsilon}\right| \leq k\right\}}} \text { a.e in } Q
$$

Letting $\varepsilon \rightarrow 0$, to obtain

$$
\eta_{n+1} \chi_{\{|u| \leq k\}}=\eta_{k} \chi_{\{|u| \leq k\}} \text { a.e in } Q-\{|u|=k\} \text { for } k \leq n \text {. }
$$

Recalling (4.81) and (4.82) allows to conclude that (4.70) holds true.

Proof of (4.71). Let $k \geq 0$ be fixed. We use the monotone character $(3.3)$ of $\mathcal{A}(x, t, \xi)$ with respest to $\xi$, to obtain

$I^{\varepsilon}=\int_{Q}\left(\mathcal{A}\left(x, t, \nabla u^{\varepsilon} \chi_{\left\{\left|u^{\varepsilon}\right| \leq k\right\}}\right)-\mathcal{A}\left(x, t, \nabla u \chi_{\{|u| \leq k\}}\right)\right)\left(\nabla u^{\varepsilon} \chi_{\left\{\left|u^{\varepsilon}\right| \leq k\right\}}-\nabla u \chi_{\{|u| \leq k\}}\right) d x d t \geq 0$.

Inequality $(4.83)$ is split into $I^{\varepsilon}=I_{1}^{\varepsilon}+I_{2}^{\varepsilon}+I_{3}^{\varepsilon}$ where

$$
\begin{aligned}
I_{1}^{\varepsilon} & =\int_{Q} \mathcal{A}\left(x, t, \nabla u^{\varepsilon} \chi_{\left\{\left|u^{\varepsilon}\right| \leq k\right\}}\right) \nabla u^{\varepsilon} \chi_{\left\{\left|u^{\varepsilon}\right| \leq k\right\}} d x d t, \\
I_{2}^{\varepsilon} & =-\int_{Q} \mathcal{A}\left(x, t, \nabla u^{\varepsilon} \chi_{\left\{\left|u^{\varepsilon}\right| \leq k\right\}}\right) \nabla u \chi_{\{|u| \leq k\}} d x d t, \\
I_{3}^{\varepsilon} & =-\int_{Q} \mathcal{A}\left(x, t, \nabla u \chi_{\{|u| \leq k\}}\right)\left(\nabla u^{\varepsilon} \chi_{\left\{\left|u^{\varepsilon}\right| \leq k\right\}}-\nabla u \chi_{\{|u| \leq k\}}\right) d x d t .
\end{aligned}
$$

We pass to the limit-sup as $\varepsilon \rightarrow 0$ in $I_{1}^{\varepsilon}, I_{2}^{\varepsilon}$ and $I_{3}^{\varepsilon}$. Let us remark that we have $u^{\varepsilon}=T_{k}\left(u^{\varepsilon}\right)$ and $\nabla u^{\varepsilon} \chi_{\left\{\left|u^{\varepsilon}\right| \leq k\right\}}=\nabla T_{k}\left(u^{\varepsilon}\right)$ a.e in $Q$, and we can assume that $k$ is such that $\chi_{\left\{\left|u^{\varepsilon}\right| \leq k\right\}}$ almost everywhere converges to $\chi_{\{|u| \leq k\}}$ (in fact this is true for almost every $k$, see Lemma 3.2 in [6]). Using (4.70), to obtain

$$
\begin{aligned}
\lim _{\varepsilon \rightarrow 0} I_{1}^{\varepsilon} & =\lim _{\varepsilon \rightarrow 0} \int_{Q} \mathcal{A}\left(x, t, \nabla u^{\varepsilon}\right) \nabla T_{k}\left(u^{\varepsilon}\right) d x d t \\
& \leq \int_{Q} \eta_{k} \nabla T_{k}(u) d x d t .
\end{aligned}
$$


In view of (4.53) and (4.55), to have

$$
\begin{aligned}
\lim _{\varepsilon \rightarrow 0} I_{2}^{\varepsilon} & =-\lim _{\varepsilon \rightarrow 0} \int_{Q} \mathcal{A}\left(x, t, \nabla u^{\varepsilon} \chi_{\left\{\left|u^{\varepsilon}\right| \leq k\right\}}\right)\left(\nabla T_{k}(u)\right) d x d t \\
& =-\int_{Q} \eta_{k}\left(\nabla T_{k}(u)\right) d x d t
\end{aligned}
$$

As a consequence of (4.53), we have for all $k>0$

$$
\lim _{\varepsilon \rightarrow 0} I_{3}^{\varepsilon}=-\int_{Q} \mathcal{A}\left(x, t, \nabla u \chi_{\{|u| \leq k\}}\right)\left(\nabla T_{k}\left(u^{\varepsilon}\right)-\nabla T_{k}(u)\right) d x d t=0 .
$$

Taking the limit-sup as $\varepsilon \rightarrow 0$ in (4.83) and using (4.84), (4.85) and (4.86) show that (4.71) holds true.

Proof of (4.72). Using (4.71) and the usual Minty argument applies it follows that (4.72) holds true.

Lemma 4.4. $\nabla T_{k}\left(v^{\varepsilon}\right)$ converges to $\nabla T_{k}(v)$ in $\left(L^{2}(Q)\right)^{N}$.

Proof. Denote $V_{\mu}^{\varepsilon}=T_{k}\left(v_{\varepsilon}\right)-\left(T_{k}(v)\right)_{\mu}$ and choose $S_{n}^{\prime}\left(v^{\varepsilon}\right)\left(T_{k}\left(v_{\varepsilon}\right)-\left(T_{k}(v)\right)_{\mu}\right)$ the test function in (4.16). One can get that

$$
\begin{aligned}
& \int_{0}^{T}\left\langle\left(B_{S}^{2}\left(v^{\varepsilon}\right)\right)_{t}, V_{\mu}^{\varepsilon}\right\rangle d t+\int_{Q} S_{n}^{\prime}\left(v^{\varepsilon}\right) \nabla v^{\varepsilon} \nabla V_{\mu}^{\varepsilon} d x d t \\
& +\int_{Q} S_{n}^{\prime \prime}\left(v^{\varepsilon}\right)\left|\nabla v^{\varepsilon}\right|^{2} V_{\mu}^{\varepsilon} d x d t=\int_{Q} f_{2}^{\varepsilon}\left(x, t, u^{\varepsilon}, v^{\varepsilon}\right) S_{n}^{\prime}\left(v^{\varepsilon}\right) V_{\mu}^{\varepsilon} d x d t .
\end{aligned}
$$

By a similar discussion, one has

$$
\begin{gathered}
\liminf _{\mu \rightarrow+\infty} \lim _{\varepsilon \rightarrow 0} \int_{0}^{T}\left\langle\left(B_{S}^{2}\left(v^{\varepsilon}\right)\right)_{t}, V_{\mu}^{\varepsilon}\right\rangle d t \geq 0 \text { for any } n \geq k, \\
\lim _{n \rightarrow+\infty \mu \rightarrow+\infty \varepsilon \rightarrow 0} \lim _{Q} \lim _{n} S_{n}^{\prime \prime}\left(v^{\varepsilon}\right)\left|\nabla v^{\varepsilon}\right|^{2} V_{\mu}^{\varepsilon} d x d t=0,
\end{gathered}
$$

and

$$
\lim _{\mu \rightarrow+\infty} \lim _{\varepsilon \rightarrow 0} \int_{Q} f_{2}^{\varepsilon}\left(x, t, u^{\varepsilon}, v^{\varepsilon}\right) S_{n}^{\prime}\left(v^{\varepsilon}\right) V_{\mu}^{\varepsilon} d x d t=0, \text { for any } n \geq 1
$$

Hence

$$
\lim _{n \rightarrow+\infty \mu \rightarrow+\infty \varepsilon \rightarrow 0} \lim _{Q} \lim _{Q} S_{n}^{\prime}\left(v^{\varepsilon}\right) \nabla v^{\varepsilon} \nabla V_{\mu}^{\varepsilon} d x d t \leq 0 .
$$

Similarly, one gets that $\nabla T_{k}\left(v^{\varepsilon}\right)$ converges to $\nabla T_{k}(v)$ in $\left(L^{2}(Q)\right)^{N}$. 
- Step 5: In this step we prove that $(u, v)$ satisfies (4.3), (4.4)-(4.7). For any fixed $n \geq 0$ one has

$$
\begin{gathered}
\int_{\left\{n \leq\left|u^{\varepsilon}\right| \leq n+1\right\}} \mathcal{A}\left(x, t, \nabla u^{\varepsilon}\right) \nabla u^{\varepsilon} d x d t \\
=\int_{Q} \mathcal{A}\left(x, t, \nabla u^{\varepsilon}\right) \nabla T_{n+1}\left(u^{\varepsilon}\right) d x d t-\int_{Q} \mathcal{A}\left(x, t, \nabla u^{\varepsilon}\right) \nabla T_{n}\left(u^{\varepsilon}\right) d x d t .
\end{gathered}
$$

According to (4.55) and (4.72) one is at liberty to pass to the limit as $\varepsilon$ tends to 0 for fixed $n \geq 1$ and to obtain

$$
\begin{aligned}
& \lim _{\varepsilon \rightarrow 0} \int_{\left\{n \leq\left|u^{\varepsilon}\right| \leq n+1\right\}} \mathcal{A}\left(x, t, \nabla u^{\varepsilon}\right) \nabla u^{\varepsilon} d x d t \\
= & \int_{Q} \mathcal{A}(x, t, \nabla u) \nabla T_{n+1}(u) d x d t-\int_{Q} \mathcal{A}(x, t, \nabla u) \nabla T_{n}(u) d x d t \\
= & \int_{\left\{n \leq\left|u^{\varepsilon}\right| \leq n+1\right\}} \mathcal{A}(x, t, \nabla u) \nabla u d x d t .
\end{aligned}
$$

Letting $n$ tends to $+\infty$ in (4.92), it follows from estimate (4.66), that

$$
\lim _{\varepsilon \rightarrow 0} \lim _{\left\{n \leq\left|u^{\varepsilon}\right| \leq n+1\right\}} \mathcal{A}\left(x, t, \nabla u^{\varepsilon}\right) \nabla u^{\varepsilon} d x d t=0 .
$$

Similarly, one can prove

$$
\lim _{\varepsilon \rightarrow 0} \lim \int_{\left\{n \leq\left|v^{\varepsilon}\right| \leq n+1\right\}}\left|\nabla v^{\varepsilon}\right|^{2} d x d t=0 .
$$

Let $S$ be a function in $W^{2, \infty}(\mathbb{R})$ such that $S^{\prime}$ has a compact. Let $k$ be a positive real number such that $\operatorname{supp}\left(S^{\prime}\right) \subset[-k, k]$. Pontwise multiplication of that approximate equation (4.15) by $\left(S^{\prime}\left(u^{\varepsilon}\right), S^{\prime}\left(v^{\varepsilon}\right)\right)$ leads to

$$
\begin{aligned}
& \left(B_{S}^{1}\left(u^{\varepsilon}\right)\right)_{t}-\operatorname{div}\left(S^{\prime}\left(u^{\varepsilon}\right) \mathcal{A}\left(x, t, \nabla u^{\varepsilon}\right)\right) \\
& +S^{\prime \prime}\left(u^{\varepsilon}\right) \mathcal{A}\left(x, t, \nabla u^{\varepsilon}\right) \nabla\left(u^{\varepsilon}\right)+\gamma\left(u^{\varepsilon}\right) S^{\prime}\left(u^{\varepsilon}\right)=f_{1}^{\varepsilon}\left(x, t, u^{\varepsilon}, v^{\varepsilon}\right) S^{\prime}\left(u^{\varepsilon}\right) \text { in } \mathcal{D}^{\prime}(Q),
\end{aligned}
$$

and

$$
\begin{aligned}
& \left(B_{S}^{2}\left(v^{\varepsilon}\right)\right)_{t}-\operatorname{div}\left(S^{\prime}\left(v^{\varepsilon}\right) \nabla v^{\varepsilon}\right) \\
& +S^{\prime \prime}\left(v^{\varepsilon}\right)\left|\nabla\left(v^{\varepsilon}\right)\right|^{2}=f_{2}^{\varepsilon}\left(x, t, u^{\varepsilon}, v^{\varepsilon}\right) S^{\prime}\left(v^{\varepsilon}\right) \text { in } \mathcal{D}^{\prime}(Q) .
\end{aligned}
$$

In what follows to pass to the limit as $\varepsilon$ tends to 0 in each term of (4.93). Since $S$ is bounded, and $\left(S\left(u^{\varepsilon}\right), S\left(v^{\varepsilon}\right)\right)$ converges to $(S(u), S(v))$ a.e in $Q$ and in $\left(L^{\infty}(Q)\right)^{2} *_{\text {-weak, then }}$ 
$\left(\left(B_{S}^{1}\left(u^{\varepsilon}\right)\right)_{t},\left(B_{S}^{2}\left(v^{\varepsilon}\right)\right)_{t}\right)$ converges to $\left(\left(B_{S}^{1}(u)\right)_{t},\left(B_{S}^{1}(v)\right)_{t}\right)$ in $\mathcal{D}^{\prime}(Q)$ as $\varepsilon$ tends to 0 . Since $\operatorname{supp}\left(S^{\prime}\right) \subset$ $[-k, k]$,

$$
S^{\prime}\left(u^{\varepsilon}\right) \mathcal{A}\left(t, x, \nabla u^{\varepsilon}\right)=S^{\prime}\left(u^{\varepsilon}\right) \mathcal{A}\left(x, t, \nabla u^{\varepsilon}\right) \chi_{\left\{\left|u^{\varepsilon}\right| \leq k\right\}} \text { a.e in } Q .
$$

The pointwise convergence of $u^{\varepsilon}$ to $u$ as $\varepsilon$ tends to 0 , the bounded character of $S$ and (4.72) of Lemma(4.3) imply that $S^{\prime}\left(u^{\varepsilon}\right) \mathcal{A}\left(x, t, \nabla u^{\varepsilon}\right)$ converges to $S^{\prime}(u) \mathcal{A}(x, t, \nabla u)$ weakly in $\left(L^{p^{\prime}(.)}(Q)\right)^{N}$ as $\varepsilon$ tends to 0 , because $S^{\prime}(u)=0$ for $|u| \geq k$ a.e in $Q$ and $S^{\prime}\left(v^{\varepsilon}\right) \nabla v^{\varepsilon}$ converges to $S^{\prime}(v) \nabla v$ weakly in $L^{2}(Q)$ as $\varepsilon$ tends to 0 . The pointwise convergence of $u^{\varepsilon}$ to $u$, the bounded character of $S^{\prime}, S^{\prime \prime}$ and (4.72) of Lemma (4.3) allow to conclude that

$$
S^{\prime \prime}\left(u^{\varepsilon}\right) \mathcal{A}\left(x, t, \nabla u^{\varepsilon}\right) \nabla T_{k}\left(u^{\varepsilon}\right) \rightarrow S^{\prime \prime}(u) \mathcal{A}(x, t, \nabla u) \nabla T_{k}(u) \text { weakly in } L^{1}(Q)
$$

as $\varepsilon \rightarrow 0$, and lemma (4.1) shows that

$$
S^{\prime \prime}\left(v^{\varepsilon}\right) \nabla^{\varepsilon} v \nabla T_{k}\left(v^{\varepsilon}\right) \rightarrow S^{\prime \prime}(v) \nabla v \nabla T_{k}(v) \text { weakly in } L^{1}(Q) .
$$

The use of (4.56) to obtain that $\gamma\left(u^{\varepsilon}\right) S^{\prime}\left(u^{\varepsilon}\right)$ converges to $\gamma(u) S^{\prime}(u)$ in $L^{1}(Q)$, and we use (4.11), (4.53) and we obtain that

$$
f_{1}^{\varepsilon}\left(x, t, u^{\varepsilon}, v^{\varepsilon}\right) S^{\prime}\left(u^{\varepsilon}\right) \text { converges to } f_{1}(x, t, u, v) S^{\prime}(u) \text { in } L^{1}(Q)
$$

and

$$
f_{2}^{\varepsilon}\left(x, t, u^{\varepsilon}, v^{\varepsilon}\right) S^{\prime}\left(v^{\varepsilon}\right) \text { converges to } f_{2}(x, t, u, v) S^{\prime}(v) \text { in } L^{1}(Q) .
$$

As a consequence of the above convergence result, the position to pass to the limit as $\varepsilon$ tends to 0 in equation (4.93) and (4.94), we conclude that $(u, v)$ satisfies (4.4) and (4.5).

It remains to show that $S(u)$ satisfies the initial condition (4.6) and $S(v)$ satisfies the initial condition (4.7). To this end, firstly remark that, $S$ being bounded, $\left(S\left(u^{\varepsilon}\right), S\left(v^{\varepsilon}\right)\right)$ is bounded in $\left(L^{\infty}(Q)\right)^{2},\left(B_{S}^{1}\left(u^{\varepsilon}\right), B_{S}^{2}\left(v^{\varepsilon}\right)\right)$ is bounded in $L^{\infty}(Q) \times L^{\infty}(Q)$. Secondly, (4.93) and (4.94), the above considerations on the behavior of the terms of this equation show that $\frac{\partial B_{S}^{1}\left(u^{\varepsilon}\right)}{\partial t}$ is bounded in $L^{1}(Q)+L^{(p-)^{\prime}}(] 0, T\left[; W^{-1, p^{\prime}(.)}(\Omega)\right)$ and $\frac{\partial B_{S}^{2}\left(v^{\varepsilon}\right)}{\partial t}$ is bounded in $L^{1}(Q)+L^{2}(] 0, T\left[; H_{0}^{1}(\Omega)\right)$. As a consequence, an Aubin's type lemma ( [20], Corollary 4) implies that $\left(B_{S}^{1}\left(u^{\varepsilon}\right), B_{S}^{2}\left(v^{\varepsilon}\right)\right)$ lies in a compact set of $\left(C(] 0, T\left[; L^{1}(\Omega)\right)\right)^{2}$. It follows that, on the one hand, $B_{S}^{1}\left(u^{\varepsilon}\right)(t=0)$ converges to $B_{S}^{1}(u)(t=0)$ strongly in $L^{1}(\Omega)$ and $B_{S}^{2}\left(v^{\varepsilon}\right)(t=0)$ converges to $B_{S}^{2}(v)(t=0)$ strongly in $L^{1}(\Omega)$. Due to (4.10), to conclude that (4.6) and (4.7) holds true. As a conclusion of Step 3 and Step 5, the proof of Theorem (4.1) is complete. 
Conflicts of Interest: The author(s) declare that there are no conflicts of interest regarding the publication of this paper.

\section{REFERENCES}

[1] Y. Akdim, J.Bennouna, M.Mekkour, H.Redwane, Existence of a Renormalised Solutions for a Class of Nonlinear Degenerated Parabolic Problems with $L^{1}$ Data, J. Partial Differ. Equ. 26 (2013), 76-98.

[2] E. Azroula, H. Redwane, M. Rhoudaf, Existence of solutions for nonlinear parabolic systems via weak convergence of truncations, Electron. J. Differ. Equ. 2010 (2010), 68.

[3] D. Blanchard, and F. Murat, Renormalised solutions of nonlinear parabolic problems with $L^{1}$ data, Existence and uniqueness, Proc. R. Soc. Edinb., Sect. A, Math. 127 (6) (1997), 1137-1152.

[4] D. Blanchard , F. Murat, and H. Redwane, Existence et unicité de la solution reormalisée d'un probléme parabolique assez général, C. R. Acad. Sci. Paris Sér., 329 (1999), 575-580.

[5] D. Blanchard, F. Murat, and H. Redwane, Existence and Uniqueness of a Renormalized Solution for a Fairly General Class of Nonlinear Parabolic Problems, J. Differ. Equ. 177 (2001), 331-374 .

[6] L. Boccardo, A. Dall'Aglio, T. Gallouët, and L. Orsina, Nonlinear parabolic equations with measure data, J. Funct. Anal., 147 (1997), 237-258 .

[7] T. M. Bendahmane, P. Wittbold, A.Zimmermann, Renormalized solutions for a nonlinear parabolic equation with variable exponents and $L^{1}$-data. J. Differ. Equ. 249 (2010), 1483-1515.

[8] M. B. Benboubker, H. Chrayteh, M. EL Moumni and H. Hjiaj, Entropy and Renormalized Solutions for Nonlinear Elliptic Problem Involving Variable Exponent and Measure Data, Acta Math. Sin. Engl. Ser. 31 (1) (2014), 151-169.

[9] Y.M. Chen, S. Levine, M. Rao, Variable exponent, linear growth functionals in image restoration, SIAM J. Appl. Math. 66 (2006) 1383-1406.

[10] Di Perna, R.-J. and P.-L. Lions, P.-L., On the Cauchy problem for Boltzmann equations : Global existence and weak stability, Ann. Math. 130 (1989), 321-366 .

[11] B. El Hamdaoui, J. Bennouna, and A. Aberqi, Renormalized Solutions for Nonlinear Parabolic Systems in the Lebesgue Sobolev Spaces with Variable Exponents, J. Math. Phys. Anal. Geom. 14 (1) (2018), 27-53.

[12] S. Fairouz, M. Messaoud, S. Kamel, Quasilinear parabolic problems in the Lebesgue-Sobolev Space with variable exponent and $L^{1}$-data. Communicated.

[13] X.L. Fan and D. Zhao, On the spaces $L^{p(x)}(U)$ and $W^{m ; p(x)}(U)$, J. Math. Anal. Appl. 263 (2001), 424-446.

[14] R. Landes, On the existence of weak solutions for quasilinear parabolic initial-boundary problems, Proc. R. Soc. Edinb., Sect. A, Math. 89 (1981), 321-366.

[15] J.-L. Lions, Quelques méthodes de résolution des problémes aux limites non linéaires. Dunod et Gauthier-Villars, 1969.

[16] S. Ouaro and A. Ouédraogo, Nonlinear parabolic equation with variable exponent and $L^{1}$ - data. Electron. J. Differ. Equ. 2017 (2017), 32.

[17] Q. Liu and Z. Guo, C. Wang, Renormalized solutions to a reaction-diffusion system applied to image denoising, Discrete Contin. Dyn. Syst., Ser. B, 21 (6) (2016), 1839-1858.

[18] H. Redwane, Existence of solution for a class of nonlinear parabolic systems, Electron. J. Qual. Theory Differ. Equ. 2007 (2007), 24.

[19] M. Sanchón, J.M. Urbano, Entropy solutions for the p(x)-Laplace equation, Trans. Amer. Math. Soc. 361 (2009), $6387-6405$.

[20] J. Simon, Compact sets in Lp(0, T;B), Ann. Mat. Pura Appl. 146 (1987), 65-96. 
[21] A. Youssef. B. Jaouad. B. Abdelkader. M. Mounir, Existence of a renormalized solution for the nonlinear parabolic systems with unbounded nonlinearities, Int. J. Res. Rev. Appl. Sci. 14 (2013), 75-89.

[22] C. Zhang, Entropy solutions for nonlinear elliptic equations with variable exponents. Electron. J. Differ. Equ. 2014 (2014), 92.

[23] C. Zhang, S. Zhou, Renormalized and entropy solution for nonlinear parabolic equations with variable exponents and $L^{1}$ data, J. Differ. Equ. 248 (2010), 1376-1400. 